\title{
First-order Dominance
}

\section{Stronger Characterization and a Bivariate Checking Algorithm}

Martin Range, Troels; Østerdal, Lars Peter

Document Version

Accepted author manuscript

Published in:

Mathematical Programming

DOI:

10.1007/s10107-017-1213-9

Publication date:

2019

\section{License}

Unspecified

Citation for published version (APA):

Martin Range, T., \& Østerdal, L. P. (2019). First-order Dominance: Stronger Characterization and a Bivariate Checking Algorithm. Mathematical Programming, 173(1/2), 193-219. https://doi.org/10.1007/s10107-017-1213-9

Link to publication in CBS Research Portal

\section{General rights}

Copyright and moral rights for the publications made accessible in the public portal are retained by the authors and/or other copyright owners and it is a condition of accessing publications that users recognise and abide by the legal requirements associated with these rights.

Take down policy

If you believe that this document breaches copyright please contact us (research.lib@cbs.dk) providing details, and we will remove access to the work immediately and investigate your claim. 


\section{First-order Dominance: Stronger Characterization and a Bivariate Checking Algorithm}

\section{Troels Martin Range and Lars Peter Osterdal}

Journal article (Accepted version*)

\section{Please cite this article as:}

Martin Range, T., \& Osterdal, L. P. (2019). First-order Dominance: Stronger Characterization and a Bivariate Checking Algorithm. Mathematical Programming, 173(1/2), 193-219. https://doi.org/10.1007/s10107-017$1213-9$

This is a post-peer-review, pre-copyedit version of an article published in Mathematical Programming. The final authenticated version is available online at:

DOl: https://doi.org/10.1007/s10107-017-1213-9

* This version of the article has been accepted for publication and undergone full peer review but has not been through the copyediting, typesetting, pagination and proofreading process, which may lead to differences between this version and the publisher's final version AKA Version of Record. 
Noname manuscript No.

(will be inserted by the editor)

\title{
First-order dominance: stronger characterization and a bivariate checking algorithm
}

\author{
Troels Martin Range • Lars Peter \\ Østerdal
}

August 25, 2017

\begin{abstract}
How to determine whether one distribution first-order dominates another is a fundamental problem that has many applications in economics, finance, probability theory, and statistics. Nevertheless, little is known about how to efficiently check first-order dominance for finite multivariate distributions. Utilizing that this problem can be formulated as a transportation problem with a special structure, we provide a stronger characterization of multivariate first-order dominance and develop a linear time complexity checking algorithm for the bivariate case. We illustrate the use of the checking algorithm when numerically assessing first-order dominance among continuous bivariate distributions.
\end{abstract}

Keywords Multivariate first-order dominance · usual stochastic order · characterization $\cdot$ network problem $\cdot$ checking algorithm

Mathematics Subject Classification (2000) 60E15 $\cdot 90 \mathrm{C} 08 \cdot 91 \mathrm{~B} 82$

Troels Martin Range (Corresponding author)

Hospital of South West Jutland and Institute of Regional Health Research: Centre of South West Jutland, University of Southern Denmark, Finsensgade 35, DK-6700 Esbjerg, Denmark E-mail: Troels.Martin.Range@rsyd.dk, Tel.: +45 2012 8140, and Department of Industrial Economics and Technology Management, Norwegian University of Science and Technology, Alfred Getz veg 3, NO-7491 Trondheim, Norway.

Lars Peter Østerdal

Department of Economics, Copenhagen Business School, Porcelænshaven 16A, 1. floor, DK2000 Frederiksberg, E-mail: lpo.eco@cbs.dk,Tel.: +45 38155641 


\section{Introduction}

The theory of stochastic dominance is a methodological cornerstone in economics, finance, probability theory, and statistics, among other fields. In welfare economics, dominance concepts are used to create partial rankings of population distributions according to either more social welfare or less inequality (e.g., Atkinson and Bourguignon [3], Gravel and Moyes [13]); in decision theory and finance, stochastic orderings are used to evaluate risky assets (e.g., Sriboonchita et al. [39]), and in statistics, appropriate order constraints can support inferences (e.g., Silvapulle and Sen [38]). For comprehensive reviews of the stochastic dominance theory we refer to Marshall and Olkin [24], Müller and Stoyan [29], and Shaked and Shanthikumar [37].

The canonical stochastic dominance concept is that of first-order dominance, also known simply as dominance or the usual (stochastic) order (e.g., Grant et al. [12], Levhari et al. [22], and Lehmann [21]). ${ }^{1}$ Intuitively, firstorder dominance means that one (dominant) distribution is superior to (i.e., provides unambiguously higher outcomes than) the other (dominated) distribution. For two multivariate finite probability mass functions, $f$ and $g, f$ first-order dominates $g$ if and only if one of the following three (equivalent) conditions hold: (a) it is possible to obtain $g$ from $f$ by moving the probability mass from better to worse outcomes, (b) the cumulative probability mass at $f$ is less than or equal to that at $g$ for every lower comprehensive subset of outcomes $^{2}$, or (c) the expected utility of $f$ is at least as high as that of $g$ for any non-decreasing utility function. ${ }^{3}$ Thus, first-order dominance is an ordinal concept that does not rely on assumptions about the relative importance

\footnotetext{
1 Less restrictive dominance criteria for better distributions have been defined by imposing stronger restrictions on the set of admissible utility functions. See, e.g., Levy and Paroush [23], Harder and Russell [15], Huang et al. [16], Atkinson and Bourguignon [3], Mosler [27], Russell and Seo [33], Scarsini [35], and Meyer and Strulovici [25].

2 A lower comprehensive subset has the defining property that if an outcome is in the subset, all smaller outcomes are also included in that subset.

3 The equivalence between (b) and (c) was proven by Lehmann [21] and Levhari et al. [22]. The equivalence between (a) and (b) can be obtained as a corollary of a theorem by Strassen [40] (see, e.g., Kamae et al. [18]) or can be established through an application of the max-flow min-cut theorem for flow networks (see, e.g., Preston [32]). Østerdal [41] provides a direct proof for equivalence between (a) and (b) in the finite case.
} 
of dimensions or the complementarity/substitutability relationships between dimensions (Arndt et al. [1]). ${ }^{4}$

For one-dimensional distributions, much research into the nature of firstorder dominance has been conducted (for example, the bibliography by Bawa [4] contains more than 400 references), and the theory is now well-developed, and with many applications. Perhaps surprisingly, little is known about how to efficiently check first-order dominance in two or more dimensions. The naive way of checking first-order dominance is to use definition (b) directly. Here, one needs to check an inequality for each lower comprehensive subset of outcomes. However, the number of inequalities to be tested grows dramatically in the total number of outcomes; therefore it is not an efficient method. ${ }^{5}$ Mosler and Scarsini [28] and Dyckerhoff and Mosler [10] describe a method based on linear programming for checking first-order dominance in the general multivariate finite case, based on definition (a) above. Empirical implementations of a method along these lines appear in Arndt et al. [1] and Arndt et al. [2]. An alternative approach would be to utilize a network flow formulation of the problem, as outlined in Preston [32] or Hansel and Troallic [14], and then check for dominance via computation of the maximum flow. We are not aware of any implementations of such a method for checking multivariate first-order dominance. Focusing on the bivariate case, we will argue below that none of these methods are efficient.

It is not difficult to observe that the problem of checking first-order dominance for finite multidimensional distributions can be formulated as a transportation problem. In this paper, we argue that this transportation problem has a special structure and allows a formulation that makes it possible to identify an upper bound on the number of diminishing transfers necessary to achieve one (dominated) distribution from another (dominant) distribution under first-order dominance. Thus, we provide a stronger characterization of first-order dominance. Furthermore, we utilize this insight to obtain a linear time complexity algorithm for the bivariate case. To our knowledge, it is faster

\footnotetext{
4 In the multivariate context, the first-order dominance concept has been used with meanings other than the one provided here. In particular, Atkinson and Bourguignon [3] and others have used the term "first-order dominance" to denote a less restrictive and easier to check dominance concept for better distributions (also known as an orthant stochastic order, cf., e.g., Dyckerhoff and Mosler [10]) which is suitable under a substitutability relationship between the dimensions.

5 Dykstra and Robertson [11] shows that for the bivariate case, with $n_{1}$ elements in the first dimension and $n_{2}$ elements in the second dimension, the number of possible lower comprehensive sets increases by $\left(\begin{array}{c}n_{1}+n_{2} \\ n_{1}\end{array}\right)$. In effect, the number increases exponentially when both dimensions grow. Sampson and Whitaker [34] extends this to the multivariate case.
} 
than any other algorithm to determine bivariate first-order dominance that has been identified in the literature. As a stepping stone to arrive at the $O(n)$ algorithm, we establish an intuitive and constructive $O\left(n^{2}\right)$ algorithm, for which we prove that a certain predefined sequence of diminishing transfers will always exist if one distribution first-order dominates another. We observe that it is necessary to obtain diminishing transfers between certain sets of outcomes, not the explicit diminishing transfers between outcomes, to prove first-order dominance. By exploiting this observation we derive the $O(n)$ algorithm.

Østerdal [41] provides a constructive proof of the relation (a) and (b) and as a result of the proof, introduces an algorithm for identifying whether one distribution first-order dominates another distribution. The method provided relies on having an initial set of transfers and then searches for potential cycles in which non-diminishing transfers are present and can be exchanged with diminishing cycles. Although it is argued that the method works, the time complexity is not considered. Indeed, the search for cycles constitutes an $O\left(n^{2}\right)$ algorithm in the verbal description of the method, and this search is executed for each potential outcome yielding an $O\left(n^{3}\right)$ algorithm. The algorithms we present in this paper for the bivariate case are significantly faster. Furthermore, Østerdal [41] proves the existence of a finite sequence of diminishing transfers without considering the number of such transfers necessary for proving firstorder dominance. In contrast, we use the transportation formulation to provide an upper bound on the number of probability mass transfers necessary to show first-order dominance. Thus, we strengthen the characterization of the equivalence (a) and (b).

It is important to use efficient methods to check multivariate first-order dominance. In applied work observations of discrete distributions with thousands or millions of levels along each dimension can be available; in this case, the efficiency of the test procedure becomes critical. When comparing continuous bivariate distributions, an approximate first-order dominance check can be performed by checking first-order dominance between corresponding discretized distributions. The more fine-grained this discretization, the better the approximation. Clearly, more efficient methods allow for more fine-grained discretizations, and thus more accurate dominance checks. ${ }^{6}$

\footnotetext{
6 Bootstrapping procedures have also been used to analyze the robustness of first-order dominance relations with respect to sample variation in the original data set (e.g., [1], [2], [17]). These procedures use random sampling with replacement requiring many first-order dominance comparisons for any pair of original distributions.
} 
The paper is organized as follows. First, in section 2, the necessary notation and basic definitions are introduced. Next, in section 3 we relate the problem of checking (multivariate) first-order dominance to the classical transportation problem. Based upon this insight, we provide a new characterization of first-order dominance for the general multivariate case. Then, we turn to the bivariate case in section 4, where we provide the two algorithms for checking dominance. We construct an example, in section 5, on how to apply the $O(n)$ algorithm to the numerical assessment of first-order dominance between continuous bivariate distributions. Section 6 concludes. All proofs are in the appendix.

\section{Notation and definitions}

An outcome is a vector $\mathbf{x}=\left(x_{1}, \ldots, x_{m}\right)$, in which each attribute $x_{j}$ is from an attribute set $X_{j}=\left\{1,2, \ldots, n_{j}\right\}, j=1, \ldots, m$, and $m \geq 2$. The outcome set is the product set $X=X_{1} \times \ldots \times X_{m}$ and has cardinality $n=\Pi_{j=1}^{m} n_{j}$. If $m=2$, we have a bivariate case. For any two elements, $\mathbf{x}, \mathbf{y} \in X$, we define $\mathbf{y} \leq \mathbf{x}$ such that $y_{j} \leq x_{j}$ for all $j$, and $\mathbf{y}<\mathbf{x}$ such that $y_{j} \leq x_{j}$ for all $j$ and $\mathbf{y} \neq \mathbf{x}$. A set $Y \subseteq X$ is called lower comprehensive if $\mathbf{x} \in Y, \mathbf{y} \in X$, and $\mathbf{y} \leq \mathbf{x}$ imply $\mathbf{y} \in Y$. An illustration of a lower comprehensive set $Y$ in the bivariate case is provided in the right portion of Figure 1.

A probability mass function is a real-valued function $f$ on $X$, such that $f(\mathbf{x}) \geq 0$ for all $\mathbf{x} \in X$ and $\sum_{\mathbf{x} \in X} f(\mathbf{x})=1$. For $\mathbf{x}, \mathbf{y} \in X$ where $\mathbf{x} \neq \mathbf{y}$, we say that a probability mass function $g$ can be obtained from a probability mass function $f$ by a transfer (of probability mass) if we have that $g(\mathbf{z})=f(\mathbf{z})$ for all $\mathbf{z} \in X \backslash\{\mathbf{x}, \mathbf{y}\}, g(\mathbf{y})=f(\mathbf{y})+\beta$, and $g(\mathbf{x})=f(\mathbf{x})-\beta$ for $\beta \in[-1,1]$. Note that if $g$ and $f$ are identical except for the values in $\mathbf{x}$ and $\mathbf{y}$, we can obtain $g$ from $f$ by transferring a suitable amount, $\beta$, of probability mass between $\mathbf{x}$ and $\mathbf{y} \cdot{ }^{7} \mathrm{~A}$ diminishing transfer (of probability mass) is a shift of probability mass from one outcome, $\mathbf{x}$, to another, $\mathbf{y}$, for $\mathbf{y}<\mathbf{x}$. A diminishing transfer for the bivariate case is shown in the left portion of Figure 1.

7 If $f(\mathbf{y}) \leq g(\mathbf{y})$ then $f(\mathbf{x}) \geq g(\mathbf{x})$ and $\beta=g(\mathbf{y})-f(\mathbf{y})=f(\mathbf{x})-g(\mathbf{x})$. Thus, the transfer we consider increases $f(\mathbf{y})$ by $\beta$ and decreases $f(\mathbf{x})$ by the same amount. The resulting probability mass function will then be identical with $g$. 

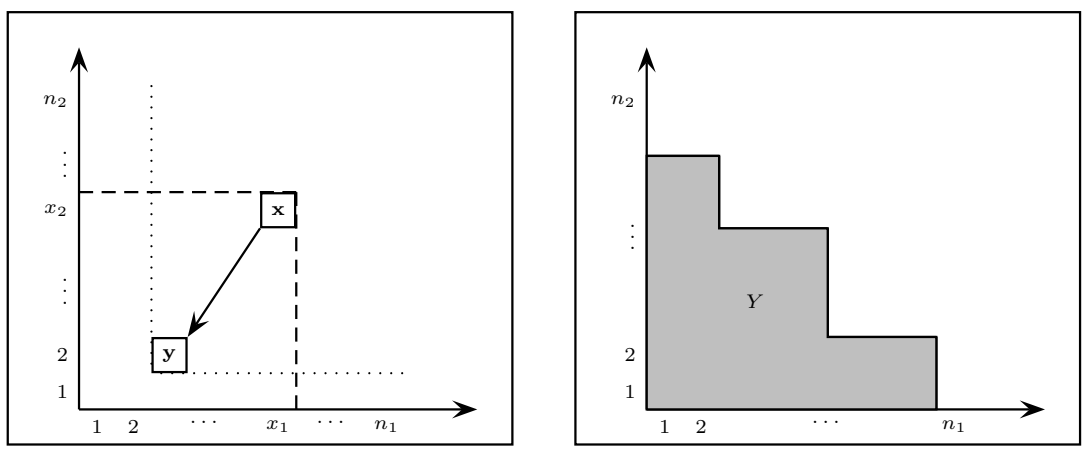

Fig. 1 Left: The lower set $L(\mathbf{x})$ is all elements below the dashed lines, the upper set $U(\mathbf{y})$ is all elements above the dotted lines, and the arrow is a diminishing transfer from $\mathbf{x}$ to $\mathbf{y}$. Right: A comprehensive set $Y$.

Let $f$ and $g$ denote two probability mass functions. A fundamental result of the stochastic dominance theory states that the following three statements are equivalent: ${ }^{8}$

(A) $g$ can be obtained from $f$ by a finite number of diminishing transfers.

(B) $\sum_{\mathbf{x} \in Y} g(\mathbf{x}) \geq \sum_{\mathbf{x} \in Y} f(\mathbf{x})$ for any lower comprehensive set $Y \subseteq X$.

(C) $\sum_{\mathbf{x} \in X} u(\mathbf{x}) f(\mathbf{x}) \geq \sum_{\mathbf{x} \in X} u(\mathbf{x}) g(\mathbf{x})$ for every non-decreasing function $u .^{9}$

We say that $f$ first-order dominates $g$ if one of these three (equivalent) properties hold.

Example 1 Let $X_{1}=\{1,2,3\}$ and $X_{2}=\{1,2,3\}$ and $f, g, h$ be bivariate probability mass functions on $X=X_{1} \times X_{2}$ as given in Figure 2. We can

\begin{tabular}{r|rrr}
$f$ & 1 & 2 & 3 \\
\hline 3 & 0.15 & 0.20 & 0.15 \\
2 & 0.05 & 0.10 & 0.12 \\
1 & 0.10 & 0.10 & 0.03
\end{tabular}

\begin{tabular}{r|rrr}
$g$ & 1 & 2 & 3 \\
\hline 3 & 0.13 & 0.07 & 0.14 \\
2 & 0.08 & 0.25 & 0.07 \\
1 & 0.15 & 0.07 & 0.04
\end{tabular}

\begin{tabular}{r|rrr}
$h$ & 1 & 2 & 3 \\
\hline 3 & 0.13 & 0.07 & 0.18 \\
2 & 0.08 & 0.18 & 0.09 \\
1 & 0.22 & 0.02 & 0.03
\end{tabular}

Fig. 2 Probability mass functions $f, g$, and $h$ for Example 1 .

observe that $f$ first-order dominates $g$ because we can obtain $g$ from $f$ by the set of diminishing probability transfers provided in Table 1 . In this table $z_{\mathbf{p r}}$ is the amount of probability mass transferred from $\mathbf{p}$ to $\mathbf{r}$, and $c_{\mathbf{p}}$ and $\rho_{\mathbf{r}}$ are variables defined in section 3.2 and 4.1 used later in the paper by Algorithm 1. Conversely, we can argue that $f$ does not first-order dominate $h$, because the

\footnotetext{
8 See footnote 3 for references to the literature establishing these equivalences.

9 A real-valued function $u$ on $X$ is non-decreasing if $\mathbf{x}, \mathbf{y} \in X$ and $\mathbf{y} \leq \mathbf{x}$ implies $u(\mathbf{y}) \leq$ $u(\mathbf{x})$.
} 


\begin{tabular}{rrrrrr} 
& from $(\mathbf{p})$ & to $(\mathbf{r})$ & amount $z_{\mathbf{p r}}$ & $c_{\mathbf{p}}$ & $\rho_{\mathbf{r}}$ \\
\hline 1 & $(1,3)$ & $(1,2)$ & 0.02 & 0 & 0.01 \\
2 & $(2,3)$ & $(1,2)$ & 0.01 & 0.12 & 0 \\
3 & $(2,3)$ & $(2,2)$ & 0.12 & 0 & 0.03 \\
4 & $(2,1)$ & $(1,1)$ & 0.03 & 0 & 0.02 \\
5 & $(3,3)$ & $(2,2)$ & 0.01 & 0 & 0.02 \\
6 & $(3,2)$ & $(2,2)$ & 0.02 & 0.03 & 0 \\
7 & $(3,2)$ & $(1,1)$ & 0.02 & 0.01 & 0 \\
8 & $(3,2)$ & $(3,1)$ & 0.01 & 0 & 0 \\
\hline
\end{tabular}

Table 1 Sequence of diminishing transfers showing that $f$ first-order dominates $g$ in Example 1.

lower comprehensive set $Y=X \backslash\{(3,3)\}$ has

$$
\sum_{\mathbf{x} \in Y} h(\mathbf{x})=0.82<0.85=\sum_{\mathbf{x} \in Y} f(\mathbf{x})
$$

therefore, we have a lower comprehensive set, violating property (B).

We add the following notation to ease the description of first-order dominance. Given an outcome $\mathbf{x} \in X$, let $L(\mathbf{x})=\{\mathbf{y} \in X \mid \mathbf{y} \leq \mathbf{x}\}$ be the lower orthant set relative to $\mathbf{x}$ and $U(\mathbf{x})=\{\mathbf{y} \in X \mid \mathbf{x} \leq \mathbf{y}\}$ be the upper orthant set relative to $\mathbf{x}$. We will refer to these sets as the lower set and the upper set, respectively. An example of both a lower set and an upper set is given in the left portion of Figure 1. For two probability mass functions, $f$ and $g$, we define the real valued function $s(\mathbf{x}):=f(\mathbf{x})-g(\mathbf{x})$ for $\mathbf{x} \in X$ along with the sets $P=\{\mathbf{x} \in X \mid s(\mathbf{x})>0\}$ and $R=\{\mathbf{x} \in X \mid s(\mathbf{x})<0\}$. For the elements of $R$, the probability mass must be added to $f$ (to become equivalent to $g$ ); similarly, for the elements of $P$, the probability mass must be subtracted from $f$ (to become equivalent to $g$ ). Thus, for $f$ to first-order dominate $g$, the elements of $P$ have excess probability mass that must be transferred to one or more elements of $R$ by diminishing transfers. More precisely, for each element $\mathbf{p} \in P$, we must transfer $s(\mathbf{p})$ probability mass to numerous elements in $L(\mathbf{p}) \cap R$. Similarly, each element $\mathbf{r} \in R$ requires $-s(\mathbf{r})$ probability mass to be transferred from numerous elements in $U(\mathbf{r}) \cap P$ for $f$ to become equivalent to $g$ by diminishing transfers.

\section{General characterizations}

As mentioned in the introduction, when checking first-order dominance it is inefficient to explicitly enumerate and check every lower comprehensive set. 
Instead, we turn to the identification of a finite number of diminishing probability mass transfers and relate this to the transportation problem.

3.1 Relation to the transportation problem

First, we must make the following observation. When using diminishing transfers to obtain $g$ from $f$ it should be noted that we can omit all the elements $\mathbf{x} \in X$ having $s(\mathbf{x})=0$. The reason is that if we transfer some probability mass from $\mathbf{x}$ to an element $\mathbf{z}$ of $L(\mathbf{x}) \backslash\{\mathbf{x}\}$, the same quantity of probability mass must be transferred to $\mathbf{x}$ from an element of $\mathbf{y} \in U(\mathbf{x}) \backslash\{\mathbf{x}\}$. By the definition of $U(\mathbf{x})$, we must have that $L(\mathbf{x}) \subset L(\mathbf{y})$, and the transfer to and from $\mathbf{x}$ can therefore be replaced by a direct transfer from $\mathbf{y}$ to $\mathbf{z}$. More generally, if a sequence of diminishing transfers uses intermediate elements of $X$, then, as previously described, it is always possible to replace one or more of these diminishing transfers with a direct diminishing transfer. Consequently, no outcome needs to both send and receive probability mass. We let $C=\{(\mathbf{p}, \mathbf{r}) \in P \times R \mid \mathbf{r} \in L(\mathbf{p})\}$ be the pairs of outcomes that correspond to possible (direct) diminishing transfers.

With the definitions given in section 2 we can formulate the problem of checking first-order dominance between two finite multivariate distributions as a bipartite network problem. ${ }^{10}$ The problem is essentially one of transportation in which the "suppliers" from $P$ must transport a required amount to the "customers" from $R$. If it is possible to transport probability mass from $\mathbf{p} \in P$ to $\mathbf{r} \in R$, i.e., $(\mathbf{p}, \mathbf{r}) \in C$, we incur a unit cost of zero for transportation from $\mathbf{p}$ to $\mathbf{r}$, whereas, if it is impossible to transport probability mass from $\mathbf{p}$ to $\mathbf{r}$, a unit cost of one is incurred for transporting probability mass from $\mathbf{p}$ to $\mathbf{r}$. Solving the resulting transportation problem either yields a zero-value objective, in which case we have identified a finite set of diminishing transfers, or a strictly positive objective showing that it is necessary to send mass from $\mathbf{p} \in P$ to a

\footnotetext{
10 Preston [32] formulates this as a maximum flow problem that can be solved in $O\left(n^{3}\right)$ by using the method described by Orlin [31]. This can, however, be improved by setting up the network in a slightly different manner. That is, instead of having an arc from $\mathbf{x}$ to all nodes in $L(\mathbf{x})$, we add arcs only from $\mathbf{x}$ to $\mathbf{y} \in L(\mathbf{x})$ having $\|\mathbf{x}-\mathbf{y}\|_{2} \leq 1$. Thus, arcs exist only from $\left(x_{1}, \ldots, x_{k}, \ldots, x_{m}\right)$ to $\left(x_{1}, \ldots, x_{k}-1, \ldots, x_{m}\right)$ for each $k=1, \ldots, m$; in other words, all nodes of the lower set that are adjacent to $\mathbf{x}$, which yields a graph with $n$ nodes and $n m$ arcs. For fixed dimension $m$, the number of arcs is then linearly bounded by the number of nodes, and we can therefore solve the corresponding max-flow problem in $O\left(n^{2} / \log n\right)$ using the method described by Orlin [31]. The linear programming based approaches described by Mosler and Scarsini [28] and Dyckerhoff and Mosler [10] are typically solved using pseudopolynomial algorithms such as the Simplex algorithm; see Schrijver [36].
} 
$\mathbf{r} \notin L(\mathbf{p}) \cap R$. Thus, if the objective is zero, we have by (A) that $f$ first-order dominates $g$, whereas if the objective is positive, $f$ does not first-order dominate $g$. If we let $b=\max \{|P|,|R|\}$ and $d=\min \{|P|,|R|\}$, and $k$ is the number of feasible connections, this problem can be solved in $O(b \log b(k+d \log d))$ using the method described by Kleinschmidt and Schannath [19]. Furthermore, there is a set-up cost of $\Theta(n)$ for identifying $P$ and $R$ from $X$ and of $O(|P||R|)$ for identifying which $\operatorname{arcs}$ are feasible. Note that $|P||R|=b d$, which is part of the algorithm's complexity. Thus, using a transportation problem algorithm directly yields a time complexity of $O(n+b \log b(k+d \log d))$.

\subsection{A stronger characterization}

Below, we present an alternative linear programming model that is based on the observation that we simply need to identify a feasible transportation problem solution with an objective value equal to zero. Let $z_{\mathbf{p r}} \geq 0$ be the amount of probability mass transferred from $\mathbf{p}$ to $\mathbf{r}$, where $(\mathbf{p}, \mathbf{r}) \in C$. Furthermore, let $c_{\mathbf{p}} \geq 0$ for $\mathbf{p} \in P$ and $d_{\mathbf{r}} \geq 0$ for $\mathbf{r} \in R$ be two sets of auxiliary variables. For given values of the $z_{\mathbf{p r}}$ variables, $c_{\mathbf{p}}$ measures the amount of probability mass that remains to be transferred out of $\mathbf{p} \in P$ to elements $\mathbf{r} \in L(\mathbf{p}) \cap R$ (to achieve $s(\mathbf{p})$ ), where $c_{\mathbf{p}}$ attains a value of zero when a sufficient amount of probability mass is transferred out of $\mathbf{p}$. Similarly, $d_{\mathbf{r}}$ measures the excess amount (compared to $-s(\mathbf{r})$ ) of probability mass transferred to element $\mathbf{r} \in R$ from the elements in $U(\mathbf{r}) \cap P$ where $d_{\mathbf{r}}$ attains a value of zero when not more than the required amount of probability mass has been transferred to $r$. Then, the problem is to identify a feasible set of transfers such that the amount of probability mass that cannot be transferred out of $\mathbf{p} \in P$ and the amount of probability mass received beyond $-s(\mathbf{r})$ for $\mathbf{r} \in R$ is minimized. Thus, we 
must solve the following linear program:

$$
\begin{aligned}
Z^{*}=\min & \sum_{\mathbf{p} \in P} c_{\mathbf{p}}+\sum_{\mathbf{r} \in R} d_{\mathbf{r}} \\
\text { s.t. } \sum_{\mathbf{r} \in L(\mathbf{p}) \cap R} z_{\mathbf{p r}}+c_{\mathbf{p}} \geq s(\mathbf{p}), & \mathbf{p} \in P \\
\sum_{\mathbf{p} \in U(\mathbf{r}) \cap P} z_{\mathbf{p r}}-d_{\mathbf{r}} \leq-s(\mathbf{r}), & \mathbf{r} \in R \\
z_{\mathbf{p r} \geq 0,} \geq & (\mathbf{p}, \mathbf{r}) \in C \\
c_{\mathbf{p} \geq 0,} & \mathbf{p} \in P \\
d_{\mathbf{r}} \geq 0, & \mathbf{r} \in R
\end{aligned}
$$

The objective (1) of this problem is to minimize both the untransferred probability mass from the elements of $P$ and the excess probability mass transferred to elements of $R$. The first constraint (2) states that each element $\mathbf{p} \in P$ either must transfer to the elements in $L(\mathbf{p}) \cap R$ or leave some of the probability mass untransferred. The second constraint (3) states that an element of $\mathbf{r}$ cannot receive more than $-s(\mathbf{r})$ probability mass from the elements in $U(\mathbf{r}) \cap P$; however, if it does, the excess probability mass is added to $d_{\mathbf{r}} \cdot{ }^{11}$ Finally, the constraints (4)-(6) simply state the non-negativity of the variables.

For the problem (1)-(6), a vector $(\mathbf{z}, \mathbf{c}, \mathbf{d})$, where $\mathbf{z}=\left(z_{\mathbf{p r}}\right)_{(\mathbf{p}, \mathbf{r}) \in C}, \mathbf{c}=$ $\left(c_{\mathbf{p}}\right)_{\mathbf{p} \in P}$ and $\mathbf{d}=\left(d_{\mathbf{r}}\right)_{\mathbf{r} \in R}$ is denoted a solution. A solution is said to be feasible if it satisfies all the constraints (2)-(6). Furthermore, a solution is said to be optimal if it is feasible and minimizes objective (1). If a solution is optimal, it is denoted $\left(\mathbf{z}^{*}, \mathbf{c}^{*}, \mathbf{d}^{*}\right)$. It can be shown that a feasible solution to (1)-(6) with $\sum_{\mathbf{p} \in P} c_{\mathbf{p}}+\sum_{\mathbf{r} \in R} d_{\mathbf{r}}=0$ has the characteristic that the inequality constraints (2) and (3) must be binding.

Our application of formulation (1)-(6) is twofold. First, we use the formulation to strengthen the characterization of first-order dominance. Specifically, we can use the relation to the transportation problem to obtain an upper bound on the number of diminishing transfers necessary to show finite (multivariate) first-order dominance. We will argue below that this upper bound corresponds to $|P|+|R|-1$. Second, in section 4, we use both this formulation and the strengthened characterization to construct efficient algorithms for checking first-order dominance in the bivariate case.

\footnotetext{
11 To be consistent we use the convention that a sum of no elements is zero, i.e., if $L(\mathbf{p}) \cap$ $R=\emptyset$, then $\sum_{\mathbf{r} \in L(\mathbf{p}) \cap R} z_{\mathbf{p r}}=0$, and if $U(\mathbf{r}) \cap P=\emptyset$, then $\sum_{\mathbf{p} \in U(\mathbf{r}) \cap P} z_{\mathbf{p r}}=0$.
} 
Clearly, if the optimal solution of problem (1)-(6) is zero, i.e., $Z^{*}=0$, we have a feasible finite series of diminishing transfers, showing that $f$ first-order dominates $g$. However, a positive objective value corresponds to the case in which no feasible series of diminishing transfers exists. In the latter case, we conclude that $f$ does not first-order dominate $g$.

Lemma $1 f$ first-order dominates $g$ if and only if a vector $\mathbf{z} \in \mathbb{R}^{|C|}$ exists with $\mathbf{z} \geq \mathbf{0}$ and

$$
\begin{aligned}
\sum_{\mathbf{r} \in L(\mathbf{p}) \cap R} z_{\mathbf{p r}}=f(\mathbf{p})-g(\mathbf{p}), \quad \forall \mathbf{p} \in P \\
\sum_{\mathbf{p} \in U(\mathbf{r}) \cap P} z_{\mathbf{p r}}=g(\mathbf{r})-f(\mathbf{r}), \quad \forall \mathbf{r} \in R
\end{aligned}
$$

It can be observed that the equations (7) and (8), in conjunction with $\mathbf{z} \geq \mathbf{0}$, describe a bounded polyhedron. Any vertex in this bounded polyhedron has at most $|P|+|R|-1$ non-zero elements, because (7) and (8) are linearly dependent. This observation leads to the following result:

Theorem $1 f$ first-order dominates $g$ if and only if $g$ can be obtained from $f$ by at most $|P|+|R|-1$ diminishing probability mass transfers.

Theorem 1 provides a sharper characterization of first-order dominance than part (A) of the equivalence. Although we can bound the necessary number of transfers to show first-order dominance, it should be noted that there are often alternative finite sequences of diminishing transfers that use more than $|P|+|R|-1$ diminishing transfers. However, the algorithms we provide in this paper will search for sets of diminishing transfers that are no larger than $|P|+|R|-1$. This search is performed by gradually, in each iteration, satisfying the amount transferred out of elements of $P$ or the amount transferred into the elements of $R$.

To the develop algorithms in the following section, another important property of the linear programming model (1)-(6) should be noted. That is, (1)-(6) may have alternative solutions yielding the same objective value. We make the following observation for a feasible solution.

Lemma 2 (Alternative solutions) Let $(\overline{\mathbf{z}}, \overline{\mathbf{c}}, \overline{\mathbf{d}})$ be a feasible solution for (1)-(6). Let $\mathbf{x}, \mathbf{y} \in P$ and $\mathbf{v}, \mathbf{w} \in L(\mathbf{x}) \cap L(\mathbf{y}) \cap R$ and put

$$
\beta=\min \left\{\bar{z}_{\mathbf{x v}}, \bar{z}_{\mathbf{y w}}\right\}
$$


If $\beta>0$, then we can construct an alternative solution $\left(\mathbf{z}^{\prime}, \mathbf{c}^{\prime}, \mathbf{d}^{\prime}\right)$ having $\mathbf{c}^{\prime}=\overline{\mathbf{c}}$, $\mathbf{d}^{\prime}=\overline{\mathbf{d}}$, and all elements of $\mathbf{z}^{\prime}$ equal to the corresponding elements of $\overline{\mathbf{z}}$ except for

$$
\begin{aligned}
& z_{\mathbf{x v}}^{\prime}=\bar{z}_{\mathbf{x v}}-\beta \\
& z_{\mathbf{y w}}^{\prime}=\bar{z}_{\mathbf{y w}}-\beta \\
& z_{\mathbf{x w}}^{\prime}=\bar{z}_{\mathbf{x w}}+\beta \\
& z_{\mathbf{y v}}^{\prime}=\bar{z}_{\mathbf{y v}}+\beta
\end{aligned}
$$

with the same objective value.

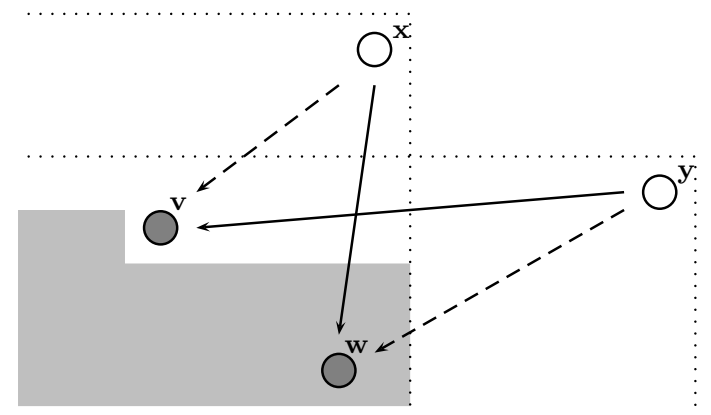

Fig. 3 Swap of probability mass transfer.

The observation from Lemma 2 is illustrated for the bivariate case in Figure 3. The full arrows are the transfers that are decreased, whereas the dashed arrows are the transfers that are increased. Lemma 2 will be used to show that given any solution to (1)-(6) with an objective value of zero we can construct an alternative solution following a specific pattern. Consequently, it will be necessary to search for this pattern only when checking first-order dominance in the bivariate case.

\section{Two algorithms for the bivariate case}

In this section, first we introduce a constructive $O\left(n^{2}\right)$ algorithm to identify a finite sequence of diminishing transfers. Then, we observe that it is not necessary to construct the finite sequence of diminishing transfers directly to show that such a sequence exists, and we use this observation as the basis for an $O(n)$ algorithm determining whether $f$ first-order dominates $g$. 
For both algorithms the elements of $X$ are traversed in a specific order. Next, we introduce the direct algorithm, and finally we introduce the indirect algorithm for checking first-order dominance.

We use two specific complete orderings of the elements of $X$. These orderings are used when iterating through the elements of $X$. We say that an element $\mathbf{x}=\left(x_{1}, x_{2}\right) \in X$ has a lower $(1,2)$-order than $\mathbf{y}=\left(y_{1}, y_{2}\right) \in X$ if $x_{1}<y_{1}$ or if $x_{1}=y_{1}$ and $x_{2}>y_{2}$. In case $\mathbf{x}$ has a lower $(1,2)$-order than $\mathbf{y}$, we write $o_{12}(\mathbf{x})<o_{12}(\mathbf{y})$ and we say that $\mathbf{y}$ has a higher $(1,2)$-order than $\mathbf{x}$. The left portion of Figure 4 shows the elements with a lower $(1,2)$-order than $\mathbf{x}$ as white and the elements with higher (1,2)-order as gray. Analogously, we say that an element $\mathbf{x}=\left(x_{1}, x_{2}\right) \in X$ has a lower $(2,1)$-order than $\mathbf{y}=\left(y_{1}, y_{2}\right) \in X$ if $x_{2}<y_{2}$ or if $x_{2}=y_{2}$ and $x_{1}>y_{1}$. In case $\mathbf{x}$ has a lower $(2,1)$-order than $\mathbf{y}$, we write $o_{21}(\mathbf{x})<o_{21}(\mathbf{y})$ and we say that $\mathbf{y}$ has a higher $(2,1)$-order than $\mathbf{x}$. The right portion of Figure 4 illustrates $(2,1)$-ordering, where the white elements have lower $(2,1)$-order than $\mathbf{x}$ and the gray elements have higher $(2,1)$-ordering than $\mathbf{x}$.
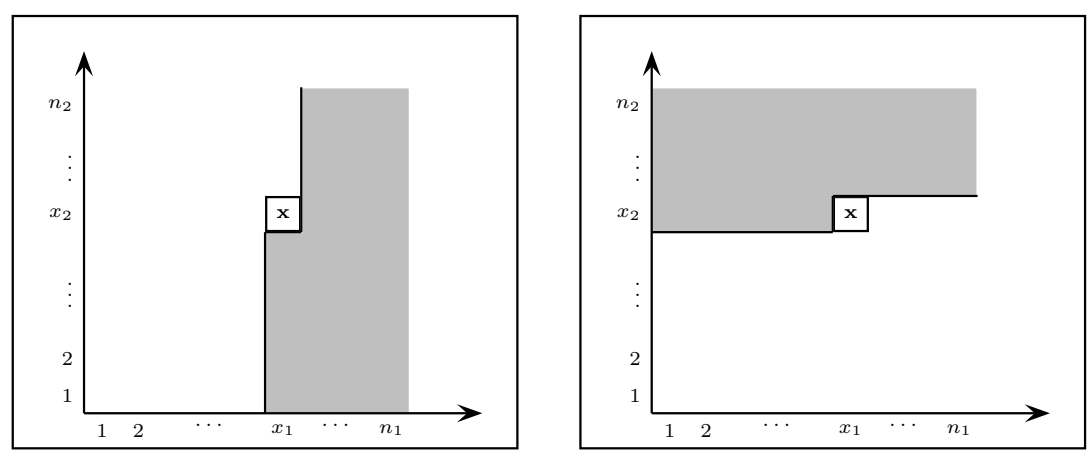

Fig. 4 Left: All gray elements have higher $(1,2)$-order than x. Right: All gray elements have a higher $(2,1)$-order than $\mathbf{x}$

Suppose that we have a subset of elements $Y \subseteq X$; given that we have an ordering $(a, b)$ either equal to $(1,2)$ or equal to $(2,1)$ then we say that an element $x \in Y$ has minimal $(a, b)$-order with respect to $Y$ if no other element $y \in Y$ exists such that $o_{a b}(y)<o_{a b}(x)$. Similarly we say that $x \in Y$ has maximal $(a, b)$-order with respect to $Y$ if no other element $y \in Y$ exists such that $o_{a b}(y)>o_{a b}(x)$. 


\subsection{A direct approach}

In the direct algorithm we explicitly identify the set of diminishing transfers by using the orderings described above. The resulting algorithm can be solved in $O\left(n^{2}\right)$ in the worst case. The algorithm identifies a set of diminishing transfers that may yield a solution value $Z^{*}$ of zero. We prove (in Lemma 3 ) that if a set of diminishing transfers yielding $Z^{*}=0$ exists for problem (1)-(6), the algorithm will identify such a set.

The idea is that we begin with a feasible, but possibly not optimal, solution to problem (1)-(6) and by carefully selecting the diminishing transfers, which should have a positive value, we gradually decrease the objective value until it is either zero, or we can show that it is not possible to obtain a value of zero.

We manipulate the variables of the formulation (1)-(6) in such a manner that we retain $d_{\mathbf{r}}=0$ for all $\mathbf{r} \in R$ while maintaining the constraint (2) binding. Thus, we have that $c_{\mathbf{p}}=s(\mathbf{p})-\sum_{\mathbf{r} \in L(\mathbf{p}) \cap R} z_{\mathbf{p r}}$ for all $\mathbf{p} \in P$, and we put $\rho_{\mathbf{r}}=-s(\mathbf{r})-\sum_{\mathbf{p} \in U(\mathbf{r}) \cap P} z_{\mathbf{p r}}$. Clearly, $\rho_{\mathbf{r}}$ corresponds to the slack variable of constraint (3) and must be non-negative. Implicitly, we initialize $z_{\mathbf{p r}}=0$ for all pairs $(\mathbf{p}, \mathbf{r}) \in P \times R .^{12}$ Thus, increasing $z_{\mathbf{p r}}$ will decrease both $c_{\mathbf{p}}$ and $\rho_{\mathbf{r}}$, and the aim is to identify a sequence of increases of $z_{\mathbf{p r}}$ such that both $c_{\mathbf{p}}$ and $\rho_{\mathbf{r}}$ become zero for all $\mathbf{p}$ and $\mathbf{r}$. If the values of $c_{\mathbf{p}}$ are zero, and because we maintain the invariant of (2) as binding, the solution found will satisfy (7). Similarly, if $\rho_{\mathbf{r}}$ is zero, and because we maintain $d_{\mathbf{r}}$ at zero, (8) is satisfied. Thus, the solution found satisfies Lemma 1 and, consequently, we have shown that $f$ first-order dominates $g$.

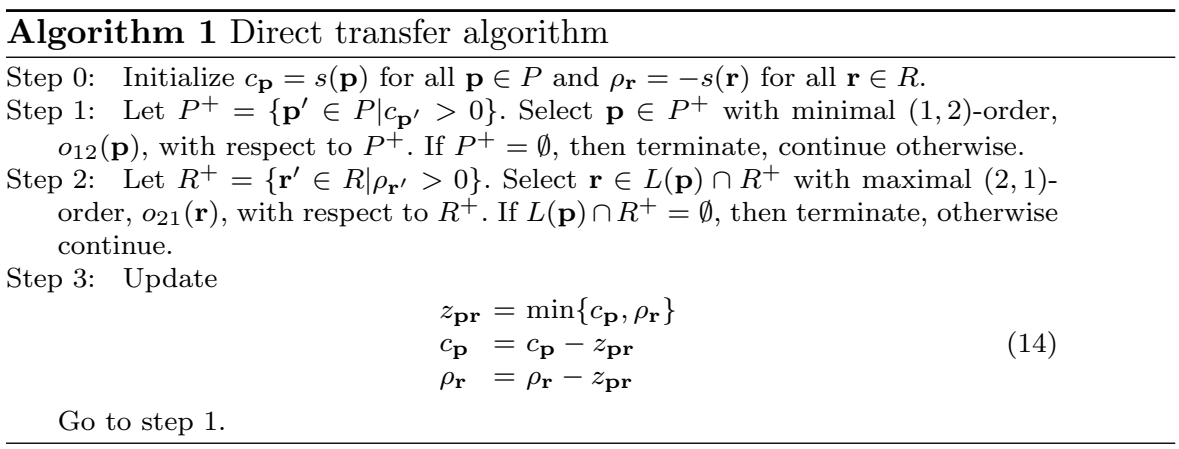

\footnotetext{
12 It is not necessary to initialize $z_{\mathbf{p r}}$ explicitly because we simply need to track which pairs $(\mathbf{p}, \mathbf{r})$ have increased values of $z_{\mathbf{p r}}$.
} 
The direct approach is provided in Algorithm 1. The algorithm initializes the variables and then repeats three steps, resulting either in a solution to the problem (1)-(6) with a value zero (terminating in step 1) or a positive objective value (terminating in step 2).

An example of the progression in the three steps is provided in the left portion of Figure 5. The element with the lowest $(1,2)$-order will be in the upper left corner, whereas the element with the highest $(1,2)$-order will be in the lower right corner of $X$. The figure shows the sequence of selections of elements of $P$ and $R$. Elements of $P$ are white nodes, whereas elements of $R$ are black nodes. The first element of $P$ encountered is $\mathbf{p}^{1}$, and it transfers probability mass to $\mathbf{r}^{6}, \mathbf{r}^{5}, \mathbf{r}^{4}$, and $\mathbf{r}^{3}$. Whereas $\mathbf{r}^{6}, \mathbf{r}^{5}$, and $\mathbf{r}^{4}$ are fully saturated, $\mathbf{r}^{3}$ received only a fraction of $-s\left(\mathbf{r}^{3}\right)$; therefore, it can receive more later in the algorithm. The sequence of diminishing transfers of probability mass away from $\mathbf{p}^{1}$ is illustrated by the full black arrows in the figure. The same is then done for $\mathbf{p}^{2}$ and $\mathbf{p}^{3}$, where the gray arrows represent the sequence of diminishing transfers from $\mathbf{p}^{2}$ and the dashed arrows show the sequence of diminishing transfers from $\mathbf{p}^{3}$.
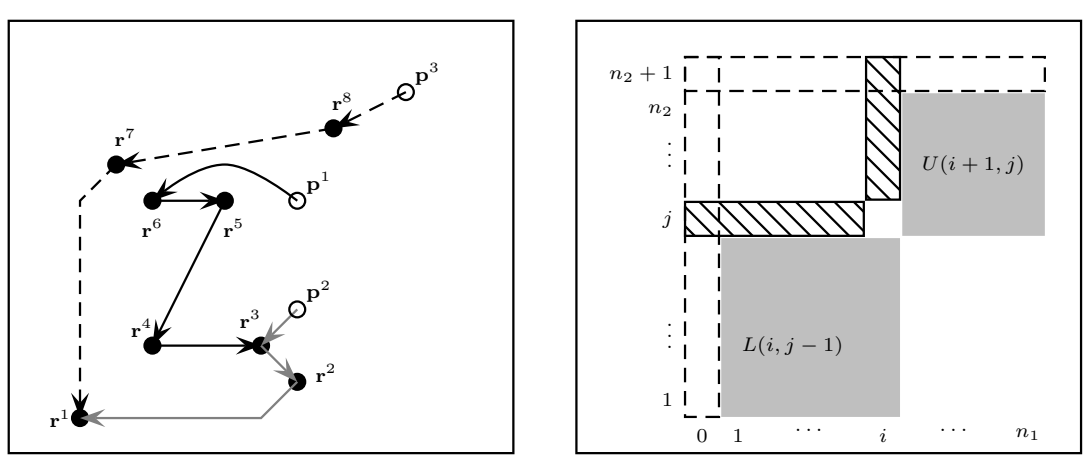

Fig. 5 Left: The process of the $O\left(n^{2}\right)$ algorithm. Right: The basic sets for the $O(n)$ algorithm.

Although $f$ clearly first-order dominates $g$ when identifying a solution satisfying Lemma 1 , it is not clear that $f$ does not first-order dominate $g$ if Algorithm 1 fails to identify a solution satisfying Lemma 1 . We can, however, show that if a set of diminishing transfers satisfying Lemma 1 exists, the sequence used in Algorithm 1 will also identify a solution satisfying Lemma 1. This finding is summarized in Lemma 3 below. 
Lemma 3 If a solution $\mathbf{z} \geq 0$ exists such that (7) and (8) are both satisfied, the sequence of diminishing transfers $\mathbf{z}^{\prime} \geq 0$ identified by Algorithm 1 will also satisfy (7) and (8).

Lemma 3 uses Lemma 2 with $\overline{\mathbf{c}}=\overline{\mathbf{d}}=0$ to transform any solution satisfying (7) and (8) into the solution obtained by Algorithm 1. Consequently, the solution found by Algorithm 1 is always an alternative solution to any solution satisfying (7) and (8). In addition, if a solution found by Algorithm 1 fails to satisfy (7) and (8), no other solution will satisfy (7) and (8). Now we can state the correctness and worst-case complexity of Algorithm 1:

Theorem 2 Algorithm 1 terminates in at most $O\left(n^{2}\right)$ iterations and either terminates with a finite sequence of diminishing transfers or shows that no such sequence exists.

A numerical example of Algorithm 1 is given in Table 1 for the two probability mass functions $f$ and $g$ in Figure 2. The sequence of the algorithm corresponds to the sequence of the entries in the table and the values of $z_{\mathbf{p r}}$, $c_{\mathbf{p}}$, and $\rho_{\mathbf{r}}$ corresponds to the values after updating in Step 3.

Algorithm 1 is an intuitive approach to checking first-order dominance. However, we can improve on the worst-case complexity of $O\left(n^{2}\right)$ to a worstcase complexity of $O(n)$ by observing that we do not always need to identify the individual diminishing transfers but only the diminishing transfers between certain sets. We will observe that we can use the same sequence as in Algorithm 1 but without performing the explicit transfers.

\subsection{An indirect approach}

We now present a more efficient algorithm for checking first-order dominance. Like Algorithm 1, this algorithm is based on iterating through the elements of $X$ in increasing $(1,2)$-order; however, it records only how much probability mass is transferred between specific aggregated subsets without specifying the transfers directly.

To ease the notation, we expand the sets $X_{1}$ and $X_{2}$ to $\bar{X}_{1}=X_{1} \cup\{0\}$ and $\bar{X}_{2}=X_{2} \cup\left\{n_{2}+1\right\}$. Now we use the simpler notation $\mathbf{x}=(i, j)$ for $i \in \bar{X}_{1}$ and $j \in \bar{X}_{2}$. Furthermore, we let $f_{i j}=f(\mathbf{x}), g_{i j}=g(\mathbf{x})$, and $s_{i j}=s(\mathbf{x})$. We let $f_{0 j}=g_{0 j}=s_{0 j}=0$ for all $j \in \bar{X}_{2}$, and $f_{i, n_{2}+1}=g_{i, n_{2}+1}=s_{i, n_{2}+1}=0$ for all $i \in \bar{X}_{1}$. We say that elements $(i, \cdot)$ are the column of $i$, and the elements $(\cdot, j)$ 
are the row of $j$. Observe the right portion of Figure 5 for an illustration of the setup, in which the dashed boxes correspond to the artificial elements added and the hatched boxes correspond to the row and column elements in row $j$ and column $i$ having a lower $(2,1)$-order and a higher (1,2)-order, respectively, than the element $(i, j)$.

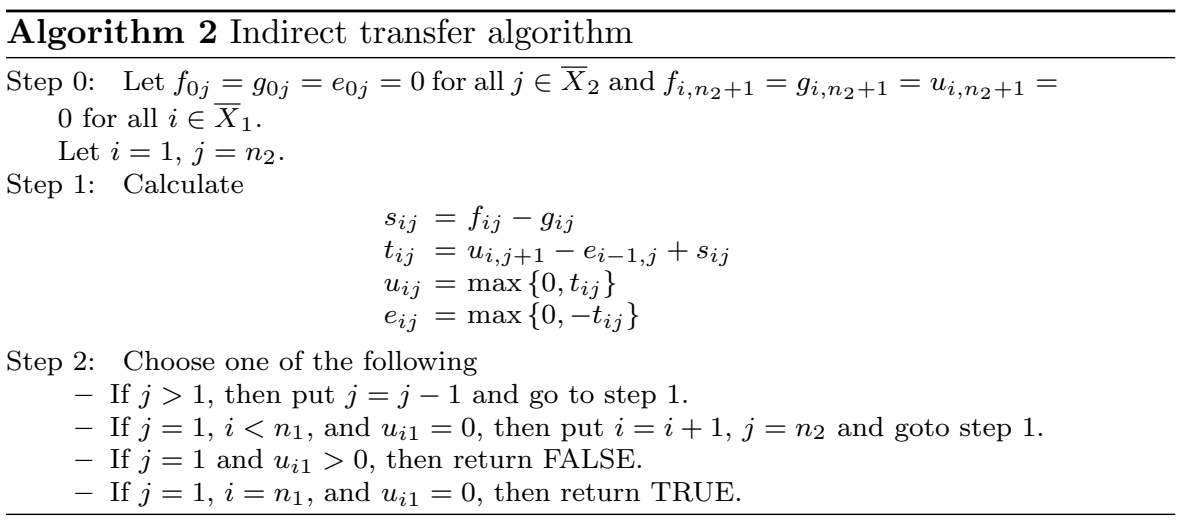

Algorithm 2 is an indirect method for determining whether $f$ first-order dominates $g$ without constructing a finite number of diminishing transfers. That is, in contrast to Algorithm 1, it does not construct a vector $\mathbf{z} \geq \mathbf{0}$ that explicitly satisfies Lemma 1. Instead Algorithm 2 identifies how much probability mass must be transferred from $S_{i j}=\{(h, k) \in X \mid h=i, k \geq j\}$ to elements of $T_{i j}=\{(h, k) \in X \mid h \leq i, k=j\}$. In the following we will describe the elements of Algorithm 2.

The algorithm iterates through $X$ in increasing $(1,2)$-order, i.e., it processes the columns in increasing order and within the columns the rows are processed in decreasing order. Consequently, at the time of processing column $i$, all columns $h<i$ have previously been processed. The reason for processing the rows within the columns in decreasing order is to transfer as much as possible to the top rows as early as possible so that it does not block transfers in later iterations. When processing row $j$ within column $i$ the algorithm has previously transferred as much as possible from elements in column $i$ with larger row indices than $j$ to rows having larger row indices than row $j$. The amount, which cannot be transferred when iterating through $X$ in $(1,2)$-order, is denoted $u_{i j}$. 
At the time of reaching element $(i, j)$ the amount $u_{i, j+1}$ remains to be transferred from some elements $(i, h)$ with $h \geq j+1$ to other elements of $L(i, j)$. Furthermore, if $s_{i j}>0$, this also must be transferred to $L(i, j)$. Again we are interested in transferring as much as possible to rows with row indices that are as large as possible. In this case it is row $j$, i.e., transferring as much of $u_{i, j+1}+s_{i j}$ to elements in row $j$ with column indices $k<i$. Elements $(k, j)$ with $k<i$ have previously been processed and we do not block additional transfers by doing this. We let $e_{i-1, j}$ be the amount that can be transferred to these row elements. Thus, if $s_{i j}>0$ then we transfer $\min \left\{u_{i, j+1}+s_{i j} ; e_{i-1, j}\right\}$ from the elements $S_{i j}$ to elements of $T_{i-1, j}$. In the case of $s_{i j}<0$, then, by an analogous argument we transfer $\min \left\{u_{i, j+1} ; e_{i-1, j}-s_{i j}\right\}$ from $S_{i, j+1}$ to $T_{i j}$. We put $t_{i j}=u_{i, j+1}-e_{i-1, j}+s_{i j}$ and show, in the proof of Lemma 4 , that $u_{i j}=\min \left\{0 ; t_{i j}\right\}$ and $e_{i j}=\min \left\{0 ;-t_{i j}\right\}$.

Example 2 (Example 1 continued) Figure 6 shows the results of applying Algorithm 2 to verify that $f$ first-order dominates $g$. Similarly, in Figure 7 we

\begin{tabular}{r|rrr}
$s$ & 1 & 2 & 3 \\
\hline 3 & 0.02 & 0.13 & 0.01 \\
2 & -0.03 & -0.15 & 0.05 \\
1 & -0.05 & 0.03 & -0.01
\end{tabular}

\begin{tabular}{r|rrr}
$u$ & 1 & 2 & 3 \\
\hline 3 & 0.02 & 0.13 & 0.01 \\
2 & 0.00 & 0.00 & 0.03 \\
1 & 0.00 & 0.00 & 0.00
\end{tabular}

\begin{tabular}{r|rrr}
$e$ & 1 & 2 & 3 \\
\hline 3 & 0.00 & 0.00 & 0.00 \\
2 & 0.01 & 0.03 & 0.00 \\
1 & 0.05 & 0.02 & 0.00
\end{tabular}

Fig. 6 Algorithm 2 testing whether $f$ first-order dominates $g$ of Example 1 .

see the results of applying Algorithm 2 to show that $f$ does not first-order dominate $h$. This result can be observed by $u_{31}=0.03>0$.

\begin{tabular}{r|rrr}
$s$ & 1 & 2 & 3 \\
\hline 3 & 0.02 & 0.13 & -0.03 \\
2 & -0.03 & -0.08 & 0.03 \\
1 & -0.12 & 0.08 & 0.00
\end{tabular}

\begin{tabular}{r|rrr}
$u$ & 1 & 2 & 3 \\
\hline 3 & 0.02 & 0.13 & 0.00 \\
2 & 0.00 & 0.04 & 0.03 \\
1 & 0.00 & 0.00 & $\mathbf{0 . 0 3}$
\end{tabular}

\begin{tabular}{r|rrr}
$e$ & 1 & 2 & 3 \\
\hline 3 & 0.00 & 0.00 & 0.03 \\
2 & 0.01 & 0.00 & 0.00 \\
1 & 0.12 & 0.00 & 0.00
\end{tabular}

Fig. 7 Algorithm 2 testing whether $f$ first-order dominates $h$ of Example 1 .

It is worth noting that the algorithm determines only how much is transferred between sets of elements. This determination does not determine the transfers between specific elements; therefore, the solution found by the algorithm represents a continuum of possible transfers, not a single possible solution.

Lemma 4 describes the case in which the algorithm returns that $f$ firstorder dominates $g$, whereas Lemma 5 describes the case in which the algorithm 
returns that $f$ does not first-order dominate $g$. Finally, Theorem 3 states the correctness and time complexity of the indirect algorithm.

Lemma 4 If Algorithm 2 terminates with $u_{n_{1} 1}=0$, a finite sequence of $d i$ minishing transfers exists such that $g$ can be obtained from $f$.

Algorithm 2 iterates through the elements in increasing $(1,2)$-order such that it starts in the top left corner and finishes in the bottom right corner. Thus, when it reaches element $\left(n_{1}, 1\right)$, the algorithm has reached the bottom right corner. If it is reached with no untransferred probability mass, we have succeeded in transferring all excess probability mass out of the elements of $P$, which is what Lemma 4 states.

Lemma 5 If Algorithm 2 terminates with $u_{i 1}>0$, a lower comprehensive set $Y \subseteq X$ exists such that $\sum_{\mathbf{x} \in Y} g(\mathbf{x})<\sum_{\mathbf{x} \in Y} f(\mathbf{x})$.

If, for $i$, we reach the bottom and have not transferred all the required probability mass from the elements of $L\left(i, n_{2}\right)$ then $u_{i 1}$ will be non-zero. Lemma 5 argues that if this occurs, we can always identify a lower comprehensive set that violates $(\mathrm{B})$ in the fundamental equivalences of first-order dominance.

Because Algorithm 2 either terminates in the situation of Lemma 4 or in the situation of Lemma 5 we can directly obtain Theorem 3.

Theorem 3 Algorithm 2 terminates in $O(n)$ iterations either stating that $f$ first-order dominates $g$ or that $f$ does not first-order dominate $g$.

It is possible to achieve the sequence of diminishing transfers without increasing the worst-case time complexity by augmenting Algorithm 2. The proof of Lemma 4 inserts elements into lists. These lists and the corresponding insertions could be added to an augmented version of Algorithm 2. The insertions and deletions of elements from the lists are performed only at the end of the lists; therefore, we can do this in $O(1)$ time complexity. Throughout the algorithm, at most $|P|+|R|$ elements are inserted into the lists because any element is inserted into one of the lists only once. Thus, the number of insertions and deletions are bounded by $|P|+|R|$ and this augmentation will have a complexity of $O(n)$.

Furthermore, it is also possible to derive a violating lower comprehensive set without increasing the worst-case time complexity by applying the constructive method in the proof of Lemma 5. The method uses no more than $\max \{|P|,|R|\}$ iterations of complexity $O(1)$; therefore, Algorithm 2 can be augmented with this construction and continue to have a worst-case time complexity of $O(n)$. 


\section{Application to numerical assessment of first-order dominance between continuous bivariate distributions}

To provide an indication of the speed and application of the indirect algorithm we have constructed a small computational example where we discretize two continuous bivariate distributions and check whether one first-order dominates the other.

We illustrate the importance of fine-grained discretization when numerically establishing whether one bivariate probability mass function dominates another. We use two truncated normalized bivariate normal distributions as an example. These distributions are depicted in Figure 8. These distributions have
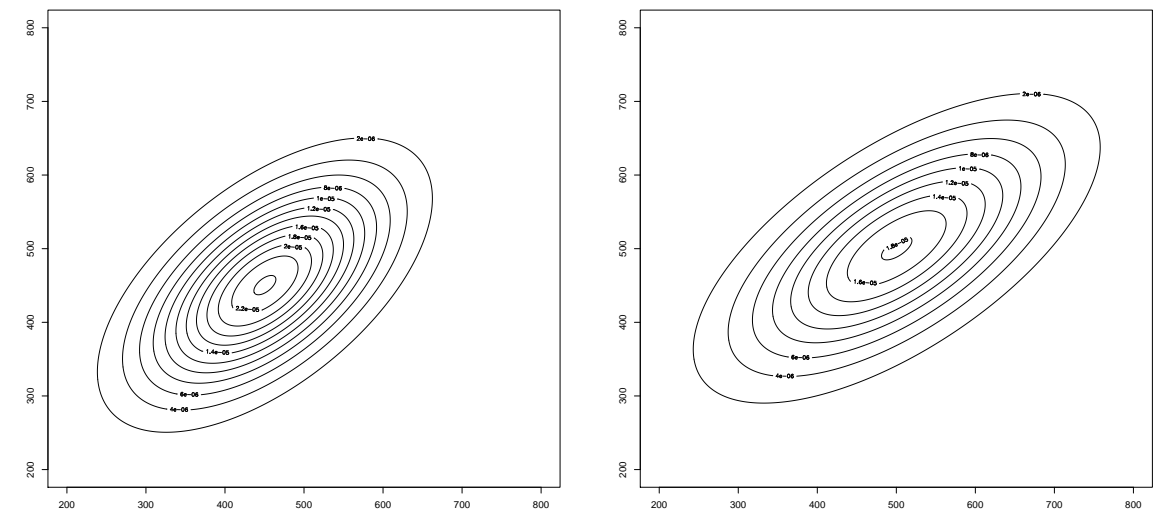

Fig. 8 Truncated normalized bivariate normal distributions, where $g$ is given to the left and $f$ is given to the right.

been constructed as follows: the distribution $f$ has mean $(500,500)$, whereas $g$ has mean $(450,450)$. These distributions have covariance matrices of

$$
\operatorname{Cov}_{f}=\left[\begin{array}{rr}
15000 & 8000 \\
8000 & 10000
\end{array}\right] \quad \operatorname{Cov}_{g}=\left[\begin{array}{l}
90005000 \\
50008000
\end{array}\right] .
$$

Using $X_{1}=\{1, \ldots, 1024\}$ and $X_{2}=\{1, \ldots, 1024\}$ we construct a discretization of each distribution by numerical integration of each discrete entry corresponding to the unit box $\left(x_{1}, x_{2}\right)$ to $\left(x_{1}+1, x_{2}+1\right) .{ }^{13}$ We truncate the value if

13 We have used the $\mathrm{R}$ package mvtnorm to obtain the probability mass in each unit box. This package uses numerical integration. 
it is less than $10 e^{-7}$. To obtain a valid distribution we normalize the outcomes by the sum of all entries. The level curves are illustrated in Figure 8.

To test the effect of the granularity of the discretization we have intervals of length $1024 / 2^{k}$ where $k=1, \ldots, 10$ yielding $2^{2 k}$ outcomes. Thus, for a unit increase in $k$, the number of outcomes is quadrupled. Thus, with a linear time complexity algorithm, we would anticipate a quadrupled time consumption.

We have implemented Algorithm 2 in $\mathrm{C}++$ and compiled it using the MinGW TDM64 5.1.0 compiler. The experiment was conducted on a single core of a computer using Microsoft Windows 7 equipped with an $\operatorname{Intel}(\mathrm{R})$ Xeon(R) CPU E5-2630 v4 @ 2.20GHz and 32Gb RAM. To measure the run time (particularly for the tests where $k$ is small) we have repeated the algorithm 1,000,000 times.

\begin{tabular}{rrr|rrr|r|c|}
$k$ & $\mathrm{I}$ & $|X|$ & $|P|$ & $|R|$ & $|C|$ & Time $(\mathrm{s})$ & $f$ dominates $g$ \\
\hline 1 & 512 & 4 & 3 & 1 & 3 & 0.016 & true \\
2 & 256 & 16 & 4 & 4 & 16 & 0.047 & true \\
3 & 128 & 64 & 12 & 9 & 95 & 0.140 & true \\
4 & 64 & 256 & 40 & 23 & 755 & 0.562 & true \\
5 & 32 & 1,024 & 120 & 83 & 8,007 & 0.750 & false \\
6 & 16 & 4,096 & 435 & 313 & 110,577 & 2.702 & false \\
7 & 8 & 16,384 & 1,644 & 1,214 & $1,618,773$ & 9.802 & false \\
8 & 4 & 65,536 & 6,389 & 4,768 & $24,717,389$ & 45.129 & false \\
9 & 2 & 262,144 & 25,154 & 18,926 & $386,544,433$ & 168.675 & false \\
10 & 1 & $1,048,576$ & 99,859 & 75,408 & $6,117,591,336$ & 636.902 & false \\
\hline
\end{tabular}

Table 2 Test of aggregated normalized bivariate truncated normal distribution

The results of the experiment are provided in Table 2. The first column provides the value of $k$ determining the interval length, as described above, and the interval length is provided in the next column, I. The resulting number of outcomes, $|X|$, is provided in the next column. The following columns $|P|$, $|R|$, and $|C|$ indicate the size of the sets of outcomes having mass to send, mass to receive, and the number of feasible send-receive pairs for diminishing transfers. Furthermore, the column Time(s) indicates how many seconds it took to execute Algorithm 2 1,000,000 times. Finally, the result of the firstorder dominance test is shown in the last column.

It is interesting to observe that different levels of aggregation yields different conclusions on whether $f$ first-order dominates $g$. For the coarse-grained levels $(k=1, \ldots, 4) f$ first-order dominates $g$; however, at the more finegrained levels $(k=5, \ldots, 10)$, the conclusion is the opposite. This finding 
demonstrates that for the numerical evaluation of continuous bivariate distributions, it is necessary to have a sufficiently high level of discretization.

The sizes $|P|$ and $|R|$ provide the number of constraints of problem (1)(6) and $|C|$ corresponds to the number of $z_{\mathbf{p r}}$ variables in the problem. In particular the number of variables will be prohibitive if one must solve the linear program directly for more fine-grained discretizations.

The time it takes to check first-order dominance is, as anticipated, approximately quadrupled when making unit increases in $k$. However, there is one exception from $k=4$ to $k=5$ reflecting that for $k=5$, the algorithm terminates as soon as it observes that $f$ does not first-order dominate $g$.

The computational time for a single instance of the finely grained discretization in which $k=10$ is on average 0.000637 seconds, i.e., a fraction of a millisecond. We consider this behavior to be fast.

If we extrapolate based on the above observation and consider the case $k=4$, in which we found dominance, and multiply the average time per instance $0.562 / 10^{6}$ by $2^{12}$ (corresponding to the increase in number of outcomes), we would have, on average, 0.002302 seconds per instance, i.e., slightly more than two milliseconds. Indeed, if we could further subdivide each of the 1024 intervals into 16 intervals and still expect a running time less than a second, i.e., we would have a discretization $16384 \times 16384$, corresponding to $k=14$, and expect to use approximately $0.562 \cdot 2^{2(k-4)} / 10^{6}=0.5892$ seconds.

\section{Final remarks}

In this paper we have obtained a strengthening of first-order dominance for the general multivariate case. Furthermore, we have described two algorithms for checking first-order dominance in the bivariate case, one of which has linear time worst-case complexity and is easy to implement. ${ }^{14}$ It should be noted that when considering the setup times, it is not possible to obtain an algorithm that has sub linear worst-case run time complexity. Thus, the indirect algorithm presented in this paper is fast.

For numerical evaluations of continuous bivariate distributions the continuous distribution can be discretized into a finite set of outcomes, as illustrated in Section 5. By checking first-order dominance of these constructed finite bivariate distributions we can obtain an approximate check on whether one

\footnotetext{
14 An implementation of Algorithm 2 in $\mathrm{C}++$ is available from the authors. For practitioners, it also easy to create a spreadsheet applying Algorithm 2.
} 
first-order dominates the other. Clearly, the more fine-grained this discretization, the better the approximation. Thus, it is important for empirical applications to have efficient methods for checking very fine-grained discretizations of continuous bivariate distributions.

The algorithms provided can easily be extended to the case of non-rectangular finite subsets of $\mathbb{R}^{2}$. This process is accomplished by extending the set of outcomes to the smallest rectangular envelopment of the given set and then putting $f(x)=g(x)=0$ for any element not contained in the original set.

It remains an open question whether it is possible to identify equally efficient (i.e., linear time worst-case complexity) algorithms in the general multivariate case. That is, algorithms that are more efficient than establishing the corresponding transportation problem or max-flow problem and solving it as such.

One might speculate that the approach suggested in this paper can be used in stochastic optimization problems with stochastic dominance constraints (see for instance Noyan and Ruszczyński [30] and Dentcheva and Ruszczyński [8]). Optimization problems containing constraints on multivariate first-order dominance may use the suggested approach. For example, Noyan and Ruszczyński [30] suggests using an integer formulation for a problem with one-dimensional first-order dominance constraints. We could augment such an approach to multivariate first-order dominance using constraints stating that if first-order dominance exists, a binary indicator should be equal to one. It would be possible to add our formulation (1)-(6) as a part of the problem and then use a big-M constraint to set the variable. Another approach would be to apply Benders' combinatorial cuts, see Codato and Fischetti [5], in which a feasibility problem (in our case problem (1)-(6) or Algorithm 2 if the problem is bivariate) is solved and if it is infeasible, a cut separating the solution is added to remove the current solution. This process may also augment the two-stage stochastic program suggested by Drapkin and Schultz [9].

A relation between first-order dominance and chance constraints exists where a target distribution must be satisfied. Dentcheva [6] provides an example in which a multiple number of chance constraints must be satisfied, yielding a target distribution. This example is equivalent to first-order dominance, and it can be augmented into a multivariate setting. Viewing the chance constraints in a first-order dominance context may improve the methods of, e.g., Dentcheva et al. [7] and Kogan and Lejeune [20] in which cuts can be separated using the Benders decomposition, as hinted above. However, this 
approach is beyond the scope of this paper; thus, we have left it for future research.

\section{Acknowledgements}

We are grateful to Bettina Klinz, the two anonymous referees, and the associate editor for valuable comments and suggestions. Furthermore, we wish to thank the participants of the 12th International Conference on Computational Management Science, 2015, in Prague and the participants of the Conference on Economic Design 2015, Istanbul, July 2015, for their helpful discussions.

\section{A Proofs}

In this appendix, the proofs of the lemmas and theorems of the paper are provided. In addition, two auxiliary lemmas, Lemma A1 and Lemma A2, are provided. The proofs are arranged in the order their respective lemmas and theorems appear in the paper except for Lemma A1 and Lemma A2 which are placed immediately prior to their use.

Lemma A1 Let $(\mathbf{z}, \mathbf{c}, \mathbf{d})$ be a feasible solution to problem (1)-(6). If $\sum_{\mathbf{p} \in P} c_{\mathbf{p}}+\sum_{\mathbf{r} \in R} d_{\mathbf{r}}=$ 0 , then

1. $c_{\mathbf{p}}=0$ for all $\mathbf{p} \in P$,

2. $d_{\mathbf{r}}=0$ for all $\mathbf{r} \in R$,

3. constraints (2) and (3) will be binding.

Proof of Lemma A1. Let (z, c, d) be a feasible solution to problem (1)-(6) with $\sum_{\mathbf{p} \in P} c_{\mathbf{p}}+$ $\sum_{\mathbf{r} \in R} d_{\mathbf{r}}=0$. As $\sum_{\mathbf{p} \in P} c_{\mathbf{p}}+\sum_{\mathbf{r} \in R} d_{\mathbf{r}}=0$, and both $\mathbf{c} \geq \mathbf{0}$ and $\mathbf{d} \geq \mathbf{0}$, we must have that $c_{\mathbf{p}}=0$ for all $\mathbf{p} \in P$ and $d_{\mathbf{r}}=0$ for all $\mathbf{r} \in R$ showing parts 1 and 2 of the lemma. Part 3 of the lemma can be realized as follows: For each pair $(\mathbf{p}, \mathbf{r}) \in C$ the variable $z_{\mathbf{p r}}$ is present in exactly one of the constraints (2), and the corresponding coefficient is equal to one. The same holds for constraint (3). Thus, we have the relation

$$
\sum_{\mathbf{p} \in P} \sum_{\mathbf{r} \in L(\mathbf{p}) \cap R} z_{\mathbf{p r}}=\sum_{\mathbf{r} \in R} \sum_{\mathbf{p} \in U(\mathbf{r}) \cap P} z_{\mathbf{p r}}
$$

Thus, summarizing constraint (2) yields the following

$$
\begin{aligned}
\sum_{\mathbf{p} \in P}\left(s(\mathbf{p})-c_{\mathbf{p}}\right) & \leq \sum_{\mathbf{p} \in P} \sum_{\mathbf{r} \in L(\mathbf{p}) \cap R} z_{\mathbf{p r}} \\
& =\sum_{\mathbf{r} \in R} \sum_{\mathbf{p} \in U(\mathbf{r}) \cap P} z_{\mathbf{p} \mathbf{r}} \\
& \leq \sum_{\mathbf{r} \in R}\left(-s(\mathbf{r})+d_{\mathbf{r}}\right) .
\end{aligned}
$$

Note that, as $\sum_{\mathbf{x} \in X} s(\mathbf{x})=\sum_{\mathbf{x} \in X} f(\mathbf{x})-\sum_{\mathbf{x} \in X} g(\mathbf{x})=0$, we have that $\sum_{\mathbf{p} \in P} s(\mathbf{p})=$ $\sum_{\mathbf{r} \in R}-s(\mathbf{r})$. Consequently, the above must hold with equality when $c_{\mathbf{p}}=0$ and $d_{\mathbf{r}}=0$. 
When $c_{\mathbf{p}}=0$ we have that $\sum_{\mathbf{p} \in P} s(\mathbf{p})=\sum_{\mathbf{p} \in P} \sum_{\mathbf{r} \in L(\mathbf{p}) \cap R} z_{\mathbf{p r}}$. Now suppose that for an element $\mathbf{p}^{\prime} \in P$ we have that $s\left(\mathbf{p}^{\prime}\right)<\sum_{\mathbf{r} \in L\left(\mathbf{p}^{\prime}\right) \cap R} z_{\mathbf{p}^{\prime} \mathbf{r}}$. Then, another $\mathbf{p}^{\prime \prime} \in P$ must exist with $s\left(\mathbf{p}^{\prime \prime}\right)>\sum_{\mathbf{r} \in L\left(\mathbf{p}^{\prime \prime}\right) \cap R} z_{\mathbf{p}^{\prime \prime} \mathbf{r}}$, i.e., requiring that $c_{\mathbf{p}^{\prime \prime}}>0$, which forces the objective to be positive. Thus, for the objective to have a zero value, we must have $s(\mathbf{p})=\sum_{\mathbf{r} \in L(\mathbf{p}) \cap R} z_{\mathbf{p r}}$ for all $\mathbf{p} \in P$. Therefore, constraint (2) must be binding for an optimal solution to have a zero value. An analogous argument can be made for (3); consequently, both constraints (2) and (3) must be binding.

Proof of Lemma 1. If $f$ first-order dominates $g$, a finite sequence of diminishing transfers exists. Consequently, a feasible set of transfers between elements of $C$ exists, yielding a solution value of zero for problem (1)-(6). Then directly by Lemma A1 we have that (7) and (8) hold. Conversely, if $\mathbf{z} \geq \mathbf{0}$ exists such that (7) and (8) hold, we have a feasible solution for problem (1)-(6) with $\mathbf{c}=\mathbf{0}$ and $\mathbf{d}=\mathbf{0}$. The values of $z_{\mathbf{p r}}$ then constitute a finite sequence of diminishing transfers.

Proof of Theorem 1. Clearly if we can obtain $f$ from $g$ by at most $|P|+|R|$ diminishing transfers, we have a finite number of diminishing transfers; consequently, we have that $f$ first-order dominates $g$.

Now assume that $f$ first-order dominates $g$. From Lemma 1, we have that the set of feasible diminishing transfers is

$$
\mathbf{Z}=\left\{\begin{array}{l|l}
\mathbf{z} \in \mathbb{R}^{|C|} \mid \begin{array}{l}
\sum_{\mathbf{r} \in L(\mathbf{p}) \cap R} z_{\mathbf{p r}}=f(\mathbf{p})-g(\mathbf{p}), \forall \mathbf{p} \in P, \\
\sum_{\mathbf{p} \in U(\mathbf{r}) \cap P} z_{\mathbf{p r}}=g(\mathbf{r})-f(\mathbf{r}), \quad \forall \mathbf{r} \in R \\
\mathbf{z} \geq \mathbf{0}
\end{array}
\end{array}\right\}
$$

and as $f$ first-order dominates $g$ we have that $\mathbf{Z} \neq \emptyset$. The set of feasible diminishing probability transfers $\mathbf{Z}$ is a convex polytope because it is the intersection of a finite number of close half spaces. Furthermore, $\mathbf{Z}$ is bounded because variables can have only non-negative values and each variable is included in at least one constraint with only positive coefficients and a positive right-hand side. If the constraint has only this variable, the variable is fixed. If the constraints have more than one variable, if we increase one variable it is necessary to decrease another variable; this can be continued only until the other variable becomes zero. Thus, $\mathbf{Z}$ is a bounded convex polytope.

Any element of $\mathbf{Z}$ corresponds to a finite number of diminishing transfers. Because $\mathbf{Z}$ is a bounded polytope we know, see, e.g., Minoux [26], that each extreme point corresponds to at least one basis dividing the variables into basic variables, which can attain positive variables, and non-basic variables, which are fixed at their lower bound of zero. The number of basic variables is equal to the number of constraints, i.e., at most $|P|+|R|$ variables can be positive. Thus, at least one element of $\mathbf{Z}$ uses at most $|P|+|R|$ positive variable values corresponding to at most $|P|+|R|$ diminishing transfers.

Finally, because of (15) we have that the constraints are linear dependent; consequently, the basic solutions will be degenerate and have at most $|P|+|R|-1$ positive variable values. Therefore at most $|P|+|R|-1$ diminishing transfers are necessary.

Proof of Lemma 2. If the two solutions have $\mathbf{c}^{\prime}=\overline{\mathbf{c}}$ and $\mathbf{d}^{\prime}=\overline{\mathbf{d}}$, they have the same objective value. Thus, we must show that altering $\overline{\mathbf{z}}$ to $\mathbf{z}^{\prime}$ maintains $\mathbf{c}^{\prime}=\overline{\mathbf{c}}$ and $\mathbf{d}^{\prime}=\overline{\mathbf{d}}$. For both $\mathbf{x}$ and $\mathbf{y}$, we have added and subtracted $\beta$ in constraint (2), thus not changing the values $c_{\mathbf{x}}$ and $c_{\mathbf{y}}$. Furthermore, for both $\mathbf{v}$ and $\mathbf{w}$, we have added and subtracted $\beta$ in 
the constraint (3) thus not changing $d_{\mathbf{v}}$ or $d_{\mathbf{w}}$ either. Consequently, because solution $\overline{\mathbf{z}}$ was feasible, $\mathbf{z}^{\prime}$ will also be, and they will have the same objective value.

Lemma A2 Let $X=X_{1} \times X_{2}$. Given four elements $\mathbf{x}, \mathbf{y}, \mathbf{v}, \mathbf{w} \in X$ having $\mathbf{w} \in L(\mathbf{x})$ and $\mathbf{v} \in L(\mathbf{y})$, then if $x_{1} \leq y_{1}$ and $v_{2} \geq w_{2}$, then $\mathbf{w} \in L(\mathbf{y})$.

Proof of Lemma A2. This result can be realized as follows: Because $\mathbf{w} \in L(\mathbf{x})$ then $w_{1} \leq x_{1}$; by assumption, we have that $x_{1} \leq y_{1}$. Furthermore, we have that $\mathbf{v} \in L(\mathbf{y})$, then $v_{2} \leq y_{2}$; by assumption, we have that $w_{2} \leq v_{2}$. Thus, $\mathbf{w} \in L(\mathbf{y})$.

Proof of Lemma 3. We show that if a $\mathbf{z} \geq \mathbf{0}$ exists satisfying (7) and (8) of Lemma 1, it can always be transformed into the solution found by Algorithm 1 while violating none of the constraints (7) and (8). Consequently, if the Algorithm 1 terminates without identifying a feasible sequence of diminishing transfers, no such sequence exists.

Note that Lemma 2 provides the possibility of shifting between alternative solutions, all satisfying the sets of equations (7) and (8). Thus, we will use this lemma for the transformation.

Because the update in step 3 the algorithm will never transfer more probability mass away from $\mathbf{p} \in P$ than $s(\mathbf{p})$ and, similarly, it will never transfer more probability mass to $\mathbf{r} \in R$ than $-s(\mathbf{r})$. Furthermore, because of the selection of pairs $(\mathbf{p}, \mathbf{r})$ in steps 1 and 2 , we guarantee that $\sum_{\mathbf{r} \in L(\mathbf{p}) \cap R} z_{\mathbf{p r}} \leq s(\mathbf{p})$ for all $\mathbf{p} \in P$ and $\sum_{\mathbf{p} \in U(\mathbf{r}) \cap P} z_{\mathbf{p r}} \leq-s(\mathbf{r})$ for all $\mathbf{r} \in R$ at any point in Algorithm 1.

Let $\mathbf{z}^{0} \geq \mathbf{0}$ satisfy equations (7) and (8) and suppose that it is different from the transfers performed by Algorithm 1. Now elements $\mathbf{p} \in P$ are selected in increasing $(1,2)-$ order and corresponding elements $\mathbf{r} \in R \cap L(p)$ in decreasing $(2,1)$-order, according to step 1 and step 2, respectively. Then, because $\mathbf{z}^{0}$ is different from the transfers made by Algorithm 1, a first pair of elements $\left(\mathbf{p}^{0}, \mathbf{r}^{0}\right)$ exists such that $z_{\left(\mathbf{p}^{0}, \mathbf{r}^{0}\right)}^{0}$ is different from what is transferred by the algorithm. Recall that Algorithm 1 is greedy and therefore transfers as much probability mass as possible as soon as possible. Thus, $z_{\left(\mathbf{p}^{0}, \mathbf{r}^{0}\right)}^{0}$ is strictly less than the amount transferred by the algorithm. Otherwise, the algorithm would have transferred more from $\mathbf{p}^{0}$ to $\mathbf{r}^{0}$. Because the algorithm can transfer more than $z_{\left(\mathbf{p}^{0}, \mathbf{r}^{0}\right)}^{0}$, we know that $z_{\left(\mathbf{p}^{0}, \mathbf{r}^{0}\right)}^{0}<s\left(\mathbf{p}^{0}\right)$ and $z_{\left(\mathbf{p}^{0}, \mathbf{r}^{0}\right)}^{0}<-s\left(\mathbf{r}^{0}\right)$. However, because $\mathbf{z}^{0}$ satisfy both equations (7) and (8) we must have that at least one $\mathbf{p}^{\prime}$ with $o_{12}\left(\mathbf{p}^{\prime}\right)>o_{12}\left(\mathbf{p}^{0}\right)$ must exist transferring $z_{\mathbf{p}^{\prime} \mathbf{r} 0}^{0}>0$ from $\mathbf{p}^{\prime}$ to $\mathbf{r}^{0}$. In the following, we let $\mathbf{p}^{\prime} \neq \mathbf{p}^{0}$ be the minimal $(1,2)$-order element of $P$, where $z_{\mathbf{p}^{\prime} \mathbf{r}^{0}}^{0}>0$. Similarly, an $\mathbf{r}^{\prime}$ with $o_{21}\left(\mathbf{r}^{\prime}\right)<o_{21}\left(\mathbf{r}^{0}\right)$ must exist transferring $z_{\mathbf{p}^{0} \mathbf{r}^{\prime}}^{0}>0$ from $\mathbf{p}^{0}$ to $\mathbf{r}^{\prime}$. We let $\mathbf{r}^{\prime} \neq \mathbf{r}^{0}$ be the maximal $(2,1)$-order element having $z_{\mathbf{p}^{0} \mathbf{r}^{\prime}}^{0}>0$.

Now observe that as $o_{12}\left(\mathbf{p}^{0}\right)<o_{12}\left(\mathbf{p}^{\prime}\right)$, we have that $p_{1}^{0} \leq p_{1}^{\prime}$. Furthermore, as $o_{21}\left(\mathbf{r}^{0}\right)>$ $o_{21}\left(\mathbf{r}^{\prime}\right)$, we have that $r_{2}^{0} \geq r_{2}^{\prime}$. Therefore, by Lemma A2 we have that $\mathbf{r}^{0}, \mathbf{r}^{\prime} \in L\left(\mathbf{p}^{0}\right) \cap L\left(\mathbf{p}^{\prime}\right) \cap$ $R$. By Lemma 2, we can put

$$
\beta=\min \left\{z_{\mathbf{p}^{\prime} \mathbf{r}^{0}}^{0}, z_{\mathbf{p}^{0} \mathbf{r}^{\prime}}^{0}\right\}
$$

and construct the new vector $\mathbf{z}^{1} \geq \mathbf{0}$ having all elements equal to $\mathbf{z}^{0}$ except for

$$
\begin{aligned}
z_{\mathbf{p}^{\prime} \mathbf{r}^{0}}^{1} & =z_{\mathbf{p}^{\prime} \mathbf{r}^{0}}^{0}-\beta \\
z_{\mathbf{p}^{0} \mathbf{r}^{\prime}}^{1} & =z_{\mathbf{p}^{0} \mathbf{r}^{\prime}}^{0}-\beta \\
z_{\mathbf{p}^{0} \mathbf{r}^{0}}^{0} & =z_{\mathbf{p}^{0} \mathbf{r}^{0}}^{1}+\beta \\
z_{\mathbf{p}^{\prime} \mathbf{r}^{\prime}}^{0} & =z_{\mathbf{p}^{\prime} \mathbf{r}^{\prime}}^{1}+\beta
\end{aligned}
$$


which satisfy (7) and (8). Consequently, $\mathbf{z}^{1}$ continues to constitutes a finite number of diminishing transfers.

By putting $\mathbf{z}^{0}=\mathbf{z}^{1}$ and repeating the argument above we will gradually obtain the transfers corresponding to those identified by Algorithm 1. Thus, if $\mathbf{z} \geq \mathbf{0}$ exists satisfying (7) and (8), it is possible to transform the vector $\mathbf{z}$ into the vector obtained by Algorithm 1 without violating (7) and (8).

Proof of Theorem 2. If Algorithm 1 terminates in step 1, a sequence of diminishing transfers has been found that satisfies all the constraints (2) and (3). This process has been conducted such that all $c_{\mathbf{p}}$ values have been decreased to zero while maintaining the $d_{\mathbf{r}}$ values at zero. Thus, a finite sequence of diminishing transfers exists.

We need to show that if Algorithm 1 terminates in step 2, no feasible sequence of diminishing transfers exists. From Lemma 3 we know that if a feasible set of diminishing transfers exists, Algorithm 1 will obtain a feasible set of diminishing transfers. Thus, if $f$ first-order dominates $g$, it is always possible to obtain a finite number of diminishing transfers by Algorithm 1; therefore, if Algorithm 1 fails to identify such a finite number of diminishing transfers, no such finite number of diminishing transfers exists.

Algorithm 1 has a time complexity of $O\left(n^{2}\right)$ because for each element $\mathbf{p} \in P$, we must search through the elements of $L(\mathbf{p})$ to identify a suitable element $\mathbf{r} \in L(\mathbf{p}) \cap R$.

Proof of Lemma 4. We prove this lemma by showing that the sequence of diminishing transfers obtained by Algorithm 1 can be obtained by Algorithm 2 as well. Algorithm 2 traverses the elements in increasing $(1,2)$-order and therefore encounters elements of $P$ in the same order as Algorithm 1. We need to track the actual transfers. Therefore, we use two sets of ordered lists which are updated during step 1 for element $(i, j)$ in Algorithm 2.

For $i \in X_{1}$ we let $\Theta_{i}=\left(\mathbf{p}^{1}, \ldots, \mathbf{p}^{k}\right)$ be the elements of $\left\{\mathbf{p} \in S_{i j} \cap P \mid c_{\mathbf{p}}>0\right\}$ ordered such that $o_{12}\left(\mathbf{p}^{a}\right)<o_{12}\left(\mathbf{p}^{a+1}\right)$ for $a=1, \ldots, k-1$. Thus, the $(1,2)$-minimal element of $\Theta_{i}$ is the first element. All elements of $\left(x_{1}, x_{2}\right) \in \Theta_{i}$ have lower $(1,2)$-order than any element of $\left(y_{1}, y_{2}\right) \in \Theta_{i+1}$ because $x_{1}=i<i+1=y_{1}$.

For $j \in X_{2}$, we let $\Delta_{j}=\left\{\mathbf{r}^{1}, \ldots, \mathbf{r}^{h}\right\}$ be the elements of $\left\{\mathbf{r} \in T_{i j} \cap R \mid \rho_{\mathbf{r}}>0\right\}$ ordered such that $o_{21}\left(\mathbf{r}^{a}\right)>o_{21}\left(\mathbf{r}^{a+1}\right)$ for $a=1, \ldots, h-1$. Thus, the first element of $\Delta_{j}$ is the $(2,1)$-maximal element of $\Delta_{j}$. Any element of $\left(x_{1}, x_{2}\right) \in \Delta_{j}$ has lower $(2,1)$-order than any element of $\left(y_{1}, y_{2}\right) \in \Delta_{j+1}$ because $x_{2}=j<j+1=y_{2}$. Thus, decreasing $j+1$ to $j$ yields lower $(2,1)$-order elements.

Step 1 of algorithm is now augmented to encompass the update of the two lists. First, if $s_{i j}>0$ then $(i, j)$ is added to the end of $\Theta_{i}$, as $(i, j) \in P$ with $c_{(i, j)}>0$ and it has higher $(1,2)$-order than the other elements of $\Theta_{i}$. Conversely, if $s_{i j}<0$ the $(i, j)$ is added to the end of $\Delta_{j}$ as it has lower $(2,1)$-order than the other elements of $\Delta_{j}$ and $(i, j) \in R$ with $\rho_{(i, j)}>0$. For simplicity we let $\tau_{i j}$ be the amount transferred from $S_{i j}$ to $T_{i j}$ and we initialize $\tau_{i j}=0$. Then, the following is repeated until either $\Theta_{i}$ is empty or $\Delta_{j}$ is empty:

1. Let $\mathbf{p}$ be the first element of $\Theta_{i}$ and $\mathbf{r}$ be the first element of $\Delta_{j}$.

2. Put

$$
\begin{aligned}
z_{\mathbf{p r}} & =\min \left\{c_{\mathbf{p}}, \rho_{\mathbf{r}}\right\} \\
c_{\mathbf{p}} & =c_{\mathbf{p}}-z_{\mathbf{p r}} \\
\rho_{\mathbf{r}} & =\rho_{\mathbf{r}}-z_{\mathbf{p r}} \\
\tau_{i j} & =\tau_{i j}+z_{\mathbf{p r}}
\end{aligned}
$$

3. If $c_{\mathbf{p}}=0$ then remove $\mathbf{p}$ from $\Theta_{i}$ and if $\rho_{\mathbf{r}}=0$ then remove $\mathbf{r}$ from $\Delta_{j}$. 
The sequence in which the pairs $(\mathbf{p}, \mathbf{r})$ are selected is equivalent to the sequence in Algorithm 1. This finding is caused by selecting $\mathbf{p}$ as the first of the elements in $\Theta_{i}$ and thereby selecting them in increasing $(1,2)$-order. Furthermore, selecting $\mathbf{r}$ as the first element of $\Delta_{j}$ corresponds to selecting the $(2,1)$-maximal element within $L(i, j) \cap R$. We also update the diminishing transfer values, $z_{\mathbf{p r}}$, in the exact same sequence as for Algorithm 1.

Finally, we must address the correspondence between the amount transferred and the values $u_{i j}$ and $e_{i j}$. We have two cases. The first case is when $s_{i j} \geq 0$. Next, the transferred amount $\tau_{i j}=\min \left\{u_{i, j+1}+s_{i j} ; e_{i-1, j}\right\}$. If $\tau_{i j}=e_{i-1, j}$ then $e_{i j}=0$ and the corresponding list $\Delta_{j}$ is empty, and $u_{i j}=u_{i, j+1}+s_{i j}-e_{i-1, j}$. Conversely, if $\tau_{i j}=u_{i, j+1}+s_{i j}$ then $u_{i j}=0$ and the corresponding list $\Theta_{i}$ is empty, and $e_{i j}=e_{i-1, j}-u_{i, j+1}-s_{i j}$. In the second case we have that $s_{i j}<0$, and the amount transferred is $\tau_{i j}=\min \left\{u_{i, j+1} ; e_{i-1, j}-s_{i j}\right\}$. If $\tau_{i j}=u_{i, j+1}$ then $u_{i j}=0$, with $\Theta_{i}$ being empty, and $e_{i j}=e_{i-1, j}-s_{i j}-u_{u, j+1}$. If $\tau_{i j}=e_{i-1, j}-s_{i j}$ then $e_{i j}=0$ and $u_{i j}=u_{i, j+1}-e_{i-1, j}+s_{i j}$. Each of the two cases corresponds to putting

$$
\begin{aligned}
& u_{i j}=\max \left\{0 ; u_{i, j+1}-e_{i-1, j}+s_{i j}\right\}=\max \left\{0 ; t_{i j}\right\} \\
& e_{i j}=\max \left\{0 ; e_{i-1, j}-u_{i, j+1}-s_{i j}\right\}=\max \left\{0 ;-t_{i j}\right\}
\end{aligned}
$$

which is exactly the values calculated in step 1 of Algorithm 2. Thus $\tau_{i j}$ provides the connection between $z_{\mathrm{pr}}$ and the two values $u_{i j}$ and $e_{i j}$.

Note that when $u_{i j}=0$ then the list $\Theta_{i}$ is empty, i.e., all elements of $\mathbf{p} \in S_{i j} \cap P$ have sent $s(\mathbf{p})$ probability mass to elements of $L(\mathbf{p}) \cap R$. Consequently, if Algorithm 2 terminates with $u_{n_{1}, 1}=0$, all lists $\Theta_{i}$, for $i \in X_{1}$, are empty, corresponding to the case in which all elements $\mathbf{p} \in P$ have sent $s(\mathbf{p})$ probability mass to elements of $L(\mathbf{p}) \cap R$. Therefore a finite sequence of diminishing transfers exists showing that $g$ can be obtained from $f$.

Proof of Lemma 5. We can explicitly identify a lower comprehensive set that violates (B) if the value of $u_{i 1}>0$ for some $i \in X_{1}$. First, note that

$$
u_{i j}-e_{i j}=\max \left\{0, t_{i j}\right\}-\max \left\{0,-t_{i j}\right\}=t_{i j}=u_{i, j+1}-e_{i-1, j}+s_{i j}
$$

and suppose that we are given a lower comprehensive set $\Upsilon$. Then, we have

$$
\sum_{(i, j) \in \Upsilon}\left(u_{i j}-e_{i j}\right)=\sum_{(i, j) \in \Upsilon}\left(u_{i, j+1}-e_{i-1, j}\right)+\sum_{(i, j) \in \Upsilon} s_{i j}
$$

Now let

$$
\begin{aligned}
& H=\left\{\quad i \in X_{1} \quad \mid \quad(i, 1) \in \Upsilon\right\} \\
& I=\left\{j \in X_{2} \quad \mid(1, j) \in \Upsilon\right\} \\
& J=\{\quad(i, j) \in \Upsilon \quad \mid \quad(i+1, j) \notin \Upsilon \quad\} \\
& K=\{\quad(i, j) \notin \Upsilon \quad \mid \quad(i, j-1) \in \Upsilon \quad\}
\end{aligned}
$$

The sets $J$ and $K$ are illustrated in Figure 9, where $\Upsilon$ is the gray area (including both shades of gray). The union of the hatched boxes corresponds to $J$, whereas the union of the dark gray boxes is the set $K$. Furthermore, the dashed box is the elements $(i, 1) \in \Upsilon$ with $i \in H$, and the dotted box is the elements $(1, j)$ with $j \in I$. We can then rearrange (18) as

$$
\sum_{(i, j) \in \Upsilon} s_{i j}=\sum_{j \in I} e_{0 j}-\sum_{(i, j) \in J} e_{i j}+\sum_{h \in H} u_{h 1}-\sum_{(i, j) \in K} u_{i j}
$$


where $\sum_{j \in I} e_{0 j}=0$ by the definition of $e_{0 j}$. Showing that the lower comprehensive set $\Upsilon$ violates condition (B) corresponds to showing that $\sum_{(i, j) \in \Upsilon} s_{i j}>0$, which is equivalent to showing that $\sum_{h \in H} u_{h 1}>\sum_{(i, j) \in J} e_{i j}+\sum_{(i, j) \in K} u_{i j}$.

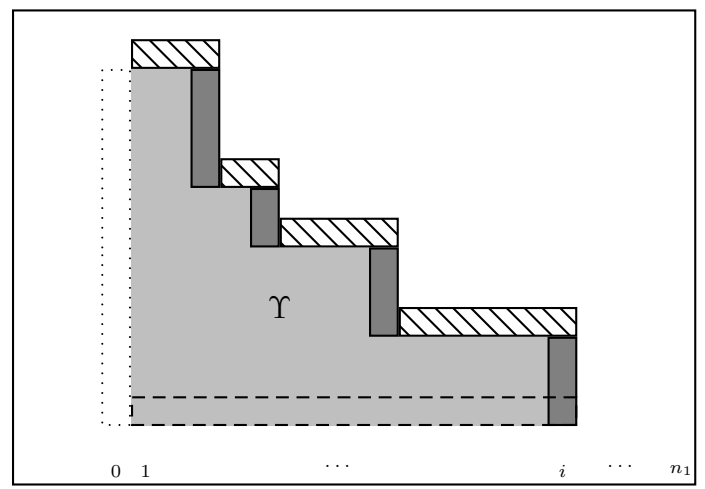

Fig. 9 A violated lower comprehensive set.

Suppose that Algorithm 2 terminates with $u_{i 1}>0$. Then, we know that $u_{h 1}=0$ for $h=1, \ldots, i-1$ and therefore $\sum_{h \in H} u_{h 1}=u_{i 1}$. Thus, if we can construct the lower comprehensive set $Y$ such that $\sum_{(h, j) \in J} e_{h j}+\sum_{(h, j) \in K} u_{h j}=0$, we have the violation we are seeking. We construct $Y$ implicitly by constructing $J$ and $K$ explicitly. Each time an element $(h, j)$ is added to $J$, all elements $(a, j)$ with $a \leq h$ are added to $Y$. Begin with $(h, j)=(i, 1)$. Because $u_{i 1}>0$, we have that $e_{i 1}=0$. Therefore add $(i, 1)$ to $J$. Repeat the following until $h=0$. If $u_{h, j+1}=0$, add $(h, j+1)$ to $K$ and put $h=h-1$, otherwise $u_{h, j+1}>0$. Consequently, $e_{h, j+1}=0$; thus, add $(h, j+1)$ to $J$ and put $j=j+1$. When terminating, we have only added elements to $J$ having $e_{h j}=0$ and elements to $K$ having elements $u_{h j}=0$, thus having $\sum_{(i, j) \in J} e_{i j}+\sum_{(i, j) \in K} u_{i j}=0<u_{i 1}$ showing that (B) is violated by $\mathrm{Y}$.

Proof of Theorem 3. If the algorithm terminates with $u_{n_{1} 1}=0$, we have by Lemma 4 and property (A) that $f$ first-order dominates $g$. Conversely, if the algorithm terminates with $u_{i 1}>0$ for $i \in X_{1}$, then by Lemma 5 a violated lower comprehensive set exists. Consequently, by property (B) $f$ does not first-order dominate $g$. Finally, because each element of $X$ is traversed maximally once and the number of operations for each element is constant, the algorithm terminates in $O(n)$ iterations.

\section{References}

1. Arndt, C., Distante, R., Hussain, M. A., Østerdal, L. P., Huong, P. L., and Ibraimo, M. (2012). Ordinal welfare comparisons with multiple discrete indicators: A first order dominance approach and application to child poverty. World Development, 40:2290 2301.

2. Arndt, C., Hussain, M., Salvucci, V., Tarp, F., and Østerdal, L. P. (2013). Advancing small area estimation. WIDER Working Paper, No. 2013/053. 
3. Atkinson, A. and Bourguignon, F. (1982). The comparison of multi-dimensioned distributions of economic status. Review of Economics Studies, 49:183-201.

4. Bawa, V. S. (1982). Stochastic dominance: A research bibliography. Management Science, 28(6):698-712.

5. Codato, G. and Fischetti, M. (2006). Combinatorial benders' cuts for mixed-integer linear programming. Operations Research, 54(4):756-766.

6. Dentcheva, D. (2006). Optimization Models with Probabilistic Constraints, pages 49-97. Springer London, London.

7. Dentcheva, D., Prékopa, A., and Ruszczyński, A. (2000). Concavity and efficient points of discrete distributions in probabilistic programming. Mathematical Programming, $89(1): 55-77$.

8. Dentcheva, D. and Ruszczyński, A. (2009). Optimization with multivariate stochastic dominance constraints. Mathematical Programming, 117(1):111-127.

9. Drapkin, D. and Schultz, R. (2010). An algorithm for stochastic programs with firstorder dominance constraints induced by linear recourse. Discrete Applied Mathematics, 158(4):291 - 297. 6th Cologne/Twente Workshop on Graphs and Combinatorial Optimization (CTW 2007).

10. Dyckerhoff, R. and Mosler, K. (1997). Orthant orderings of discrete random vectors. Journal of Statistical Planning and Inference, 62:193-205.

11. Dykstra, R. L. and Robertson, T. (1982). An algorithm for isotonic regression for two or more independent variables. The Annals of Statistics, 10(3):pp. 708-716.

12. Grant, S., Kajii, A., and Polak, B. (1992). Many good choice axioms: when can manygood lotteries be treated as money lotteries? Journal of Economic Theory, 56:313-337.

13. Gravel, N. and Moyes, P. (2012). Ethically robust comparisons of bidimensional distributions with an ordinal attribute. Journal of Economic Theory, 147:1384-1426.

14. Hansel, G. and Troallic, J. (1978). Measures marginales et théorème de Ford-Fulkerson. Zeitschrift für Wahrsheinlichkeitstheorie und verwandte Gebiete, 43:245-251.

15. Harder, J. and Russell, W. (1974). Stochastic dominance in choice under uncertainty. In Balch, M., D.L., M., and Wu, S., editors, Essays on Economic Behavior under Uncertainty. North-Holland, Amsterdam.

16. Huang, C., Kira, D., and Vertinsky, I. (1978). Stochastic dominance rules for multiattribute utility functions. Review of Economic Studies, 45:611-615.

17. Hussain, M. A., Jørgensen, M. M., and Østerdal, L. (2014). Refining population health comparisons: A multidimensional first order dominance approach. COHERE Working Paper, University of Southern Denmark.

18. Kamae, T., Krengel, U., and O'Brien, G. (1977). Stochastic inequalities on partially ordered spaces. The Annals of Probability, 5:899-912.

19. Kleinschmidt, P. and Schannath, H. (1995). A strongly polynomial algorithm for the transportation problem. Mathematical Programming, 68:1-13.

20. Kogan, A. and Lejeune, M. A. (2014). Threshold boolean form for joint probabilistic constraints with random technology matrix. Mathematical Programming, 147(1):391-427.

21. Lehmann, E. (1955). Ordered families of distributions. The Annals of Mathematical Statistics, 26:399-419.

22. Levhari, D., Paroush, J., and Peleg, B. (1975). Efficiency analysis for multivariate distributions. Review of Economic Studies, 42:87-91. 
23. Levy, H. and Paroush, J. (1974). Toward multivariate efficiency criteria. Journal of Economic Theory, 7:129-142.

24. Marshall, A. and Olkin, I. (1979). Inequalitites: Theory of Majorization and Its Applications, volume 143 of Mathematics in Science and Engineering. New York: Academic Press.

25. Meyer, M. and Strulovici, B. (2015). Beyond correlation: Measuring interdependence through complementarities. Unpublished working paper.

26. Minoux, M. (1986). Mathematical programming: theory and algorithms. WileyInterscience series in discrete mathematics and optimization. Wiley.

27. Mosler, K. (1984). Stochastic dominance decision rules when the attributes are utility independent. Management Science, 30:1311-1322.

28. Mosler, K. and Scarsini, M. (1991). Some theory of stochastic dominance. In Mosler, K. and Scarsini, M., editors, Stochastic Orders and Decision under Risk. Institute of Mathematical Statistics, Heyward, California.

29. Müller, A. and Stoyan, D. (2002). Comparison Methods for Stochastic Models and Risks. John Wiley and Sons.

30. Noyan, N. and Ruszczyński, A. (2008). Valid inequalities and restrictions for stochastic programming problems with first order stochastic dominance constraints. Mathematical Programming, 114(2):249-275.

31. Orlin, J. B. (2013). Max flows in o(nm) time, or better. In Proceedings of the 45th Annual ACM Symposium on Symposium on Theory of Computing, STOC '13, pages 765-774, New York, NY, USA. ACM.

32. Preston, C. (1974). A generalization of the FKG inequalities. Communications in Mathematical Physics.

33. Russell, W. and Seo, T. (1978). Ordering uncertain prospects: The multivariate utility functions case. Review of Economic Studies, 45:605-610.

34. Sampson, A. R. and Whitaker, L. R. (1988). Positive dependence, upper sets, and multidimensional partitions. Mathematics of Operations Research, 13(2):pp. 254-264.

35. Scarsini, M. (1988). Dominance conditions for multivariate utility functions. Management Science, 34:454-460.

36. Schrijver, A. (1987). Theory of Linear and Integer Programming. Discrete Mathematics and Optimization. Wiley-Interscience.

37. Shaked, M. and Shanthikumar, J. (2007). Stochastic Orders. Springer.

38. Silvapulle, M. J. and Sen, P. K. (2011). Constrained statistical inference: Order, inequality, and shape constraints., volume 912. Wiley-Interscience.

39. Sriboonchita, S., Dhompongsa, S., Wong, W. K., and Nguyen, H. T. (2009). Stochastic dominance and applications to finance, risk and economics. Chapman \& Hall/CRC.

40. Strassen, V. (1965). The existence of probability measures with given marginals. The Annals of Mathematical Statistics, 36:423439.

41. Østerdal, L. P. (2010). The mass transfer approach to multivariate discrete first order stochastic dominance: direct proof and implications. Journal of Mathematical Economics, 46:1222-1228. 